\title{
Consistent population activity on the scale of minutes in the mouse hippocampus
}

\author{
Yue Liu ${ }^{1}$, Samuel Levy, William Mau \\ Boston University \\ Nitzan Geva, Alon Rubin, Yaniv Ziv \\ Weizmann Institute of Science \\ Michael E. Hasselmo, Marc W. Howard \\ Boston University
}

\begin{abstract}
Neurons in the hippocampus fire in consistent sequence over the timescale of seconds during the delay period of some memory experiments. For longer timescales, firing of hippocampal neurons also changes slowly over minutes within experimental sessions. It was thought that these slow dynamics are caused by stochastic drift or a continuous change in the representation of the episode, rather than consistent sequences unfolding over minutes. This paper studies the consistency of contextual drift in three chronic calcium imaging recordings from the hippocampus CA1 region in mice. Computational measures of consistency show reliable sequences within experimental trials at the scale of seconds as one would expect from time cells or place cells during the trial, as well as across experimental trials on the scale of minutes within a recording session. Consistent sequences in the hippocampus are observed over a wide range of time scales, from seconds to minutes. Hippocampal activity could reflect a scale-invariant spatiotemporal context as suggested by theories of memory from cognitive psychology.
\end{abstract}

When we remember a particular experience from a trip, other memories from the same trip would also come into mind. Indeed, the retrieval of an episodic memory is believed to involve recovery of the spatiotemporal context associated with that particular episode (Tulving, 1983). The hippocampus has long been implicated in episodic memory (Scoville \& Milner, 1957) and it contains single neurons that are active when the animal is at a particular location within an environment (O'Keefe \& Dostrovsky, 1971; Moser, Kropff, \& Moser, 2008) or at a particular time point during the gap between two stimuli (Pastalkova, Itskov, Amarasingham, \& Buzsaki, 2008; MacDonald, Lepage, Eden, \& Eichenbaum, 2010; Kraus, Robinson, White, Eichenbaum, \& Hasselmo, 2013) (Figure 1a, top). Taken together, this neural population activity can be thought of as a state of spatiotemporal context

\footnotetext{
${ }^{1}$ Current address: Center for Neural Science, New York University. liuyue@nyu.edu
} 
upon which memories are organized (O'Keefe \& Nadel, 1978; Howard, Fotedar, Datey, \& Hasselmo, 2005; Polyn \& Kahana, 2008; Staresina \& Davachi, 2009; Hasselmo, 2012; Eichenbaum, 2017; DuBrow, Rouhani, Niv, \& Norman, 2017; Buzsáki \& Tingley, 2018; Ekstrom \& Ranganath, 2018; Yonelinas, Ranganath, Ekstrom, \& Wiltgen, 2019).

Episodic memory retrieval is organized according to spatiotemporal proximity at many different scales. When a participant has an episodic memory for an event from a particular temporal position within a list (Kahana, 1996) or spatial position within an environment (Miller, Lazarus, Polyn, \& Kahana, 2013), this brings to mind events from nearby positions, in time or in space. If episodic memory is indeed associated with the recovery of a spatiotemporal context (Tulving, 1983), then the effect of proximity on behavior could be caused by gradual changes in spatiotemporal context reflected in hippocampal ensembles (Manns, Howard, \& Eichenbaum, 2007; Ezzyat \& Davachi, 2014; Rubin, Geva, Sheintuch, \& Ziv, 2015; Cai et al., 2016). In this view, memories for events close in space or time are linked because of overlap in the spatiotemporal contexts associated with those events. Sequences of time cells or place cells could serve as a spatiotemporal context; because they change slowly over time they could mediate associations between nearby events (Wallenstein, Eichenbaum, \& Hasselmo, 1998; Howard et al., 2005; Hsieh, Gruber, Jenkins, \& Ranganath, 2014). Thus far, time cell sequences have only been observed over a few seconds within the delay period of an experimental trial embedded in a much longer recording session (Pastalkova et al., 2008; MacDonald, Lepage, Eden, \& Eichenbaum, 2011; Kraus et al., 2013). However, behavioral effects linking memories separated in time are observed over many timescales in list learning experiments (Howard, Youker, \& Venkatadass, 2008; Unsworth, 2008) and can span days and weeks in memory for real-world events (Healey, Long, \& Kahana, 2019; Uitvlugt \& Healey, 2019). If memories across lists separated by many minutes can be linked, this suggests that hippocampal sequences should also unfold across trials over the scale of minutes. Perhaps the sequence of cells that unfolds in the moments following the beginning of a delay period of a few seconds has an analog in a sequence that unfolds over the entire recording session following the beginning of the session.

A series of studies have found that the activity of hippocampal neurons does change slowly over long timescales. For example, it was reported that population neuronal activity in CA1 exhibits gradual changes over multiple trials that span minutes (Manns et al., 2007; Ziv et al., 2013; Mau et al., 2018) (Figure 1a, bottom). It has also been reported that place cell and time cell activity slowly "drift" across hours and days (Ziv et al., 2013; Mankin, Diehl, Sparks, Leutgeb, \& Leutgeb, 2015; Mankin et al., 2012; Rubin et al., 2015; Mau et al., 2018; Cai et al., 2016). The observation of these slow changes with multiple recording techniques make it unlikely that they are a recording artifact. However, it is possible that this slow drift is simply caused by stochastic processes in the neural system or perhaps a gradual but continuous change in the representation of events. Stochastic mechanisms would cause changes in firing across trials but there is no reason to expect that they would cause the same sequence over repeated experiences (Figure 1b, bottom left). However, if slow changes are generated by the same mechanism as time cell sequences, we would expect the dynamics to be consistent across repeated experiences. In much the same way as time cell sequences can be understood as coding for the time since the delay period began, slow changes in firing across trials could contain information about the time since the recording session began. 
Figure 1 describes a strategy for data analyses to distinguish consistent sequences from stochastic drifts. Consider a population of cells being recorded over two separate experiences. During each experience the activity of the population changes gradually. This effect can be demonstrated by measuring the correlation of firing over the population at different points in time. As one chooses time points further apart from one another within the experience the population becomes more decorrelated. Now, suppose that the population fires consistently from one experience to the next (Figure 1b, top left). In this case one would observe an analogous decorrelation when examining firing across different experiences (Figure 1b, top right). Although the two experiences could be separated by a time interval much longer than the duration of the experience itself, the population activity from a particular time point in each experience will be similar even if those time points are taken from different experiences. In contrast, if the within-experience correlation were not due to consistent sequences but, say, stochastic variability (Figure 1b, bottom left), the population would still change gradually over time within one experience. However, if the population activity simply decorrelates with time, that would also mean that one would not observe correlation between analogous time points within different trials (Figure 1b, bottom right).

The strategy of this paper is to evaluate whether slow changes in hippocampal activity across trials include consistent sequences extending across multiple trials. To compare to more well-understood sequences - time cells and place cells - we apply the same analyses to population activity both within trials and across trials. It is impossible to assess whether a sequence is consistent or not if one cannot record from the same population. Therefore, we study populations recorded using chronic calcium imaging technique that allow identification of the same neurons across recording sessions (Ziv et al., 2013). We found that across three behavioral tasks, populations of neurons in the CA1 region of mouse hippocampus exhibit consistent dynamics both within seconds-long trials and across trials, spanning many minutes within a recording session.

\section{Results}

To distinguish consistent sequences from stochastic dynamics (Figure 1), we examined the consistency of the neuronal dynamics across two timescales while mice performed reward-based navigational tasks (Mau et al., 2018; Levy, Kinsky, Mau, Sullivan, \& Hasselmo, 2019; Rubin et al., 2015). In all experiments, each session consists of multiple trials, during which mice were trained to navigate through an environment to obtain rewards (Figure 2). One-photon endoscope calcium imaging was used to record the activity of many neurons in the CA1 region of the hippocampus across sessions. Images from different sessions were cross-registered so that the activity of the same neurons could be tracked across sessions (Methods). We develop a series of computational measures for the consistency of activity over seconds-long delay intervals across trials. Not surprisingly these measures of consistency detect sequences over seconds that are consistent across trials, driven by time cells and place cells. Next, we apply the same computational measures to detect slow sequences of activity over multiple trials that are consistent across experimental sessions. To the extent these measures show the same kind of consistent dynamics described by time cells and place cells, we will establish that hippocampal ensembles exhibit consistent sequences of activity across trials at the time scale of a session. 
a.
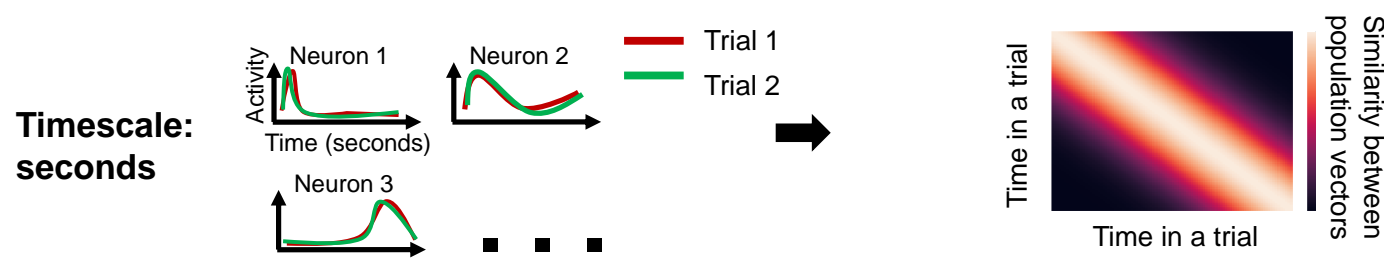

Timescale: Minutes
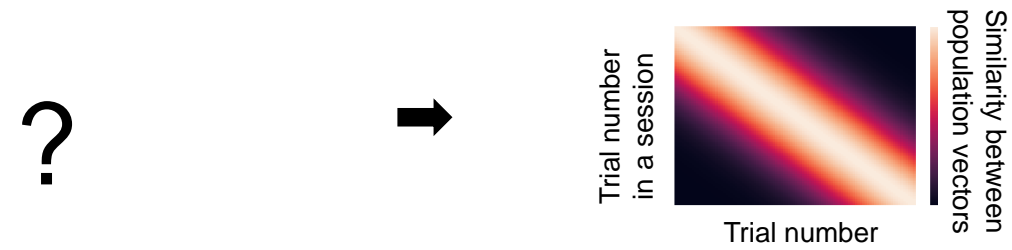

in a session

b.
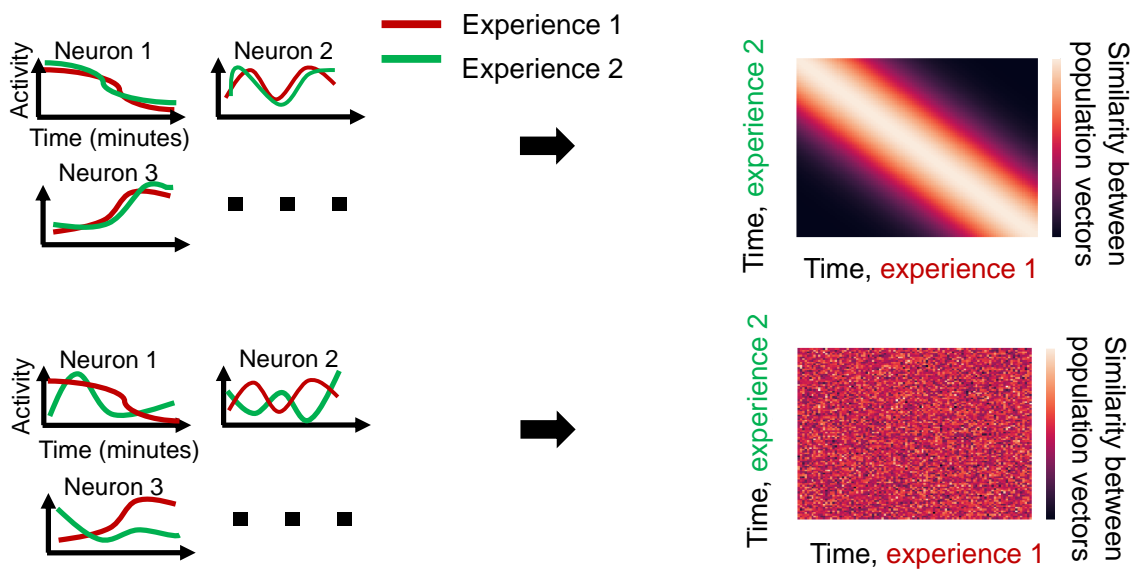

Figure 1. Distinguishing slow consistent sequences from random drifts. a. Top: The firing of time cells changes across seconds in sequences that are consistent across trials (left), which contributes to the decorrelation of population activity pattern over the timescale of seconds (right). Bottom: The firing of hippocampal neurons also changes slowly over trials (right), but it is not known if this is driven by consistent sequences on the timescale of minutes (left). b. Two possibilities for the nature of the slow dynamics over minutes. Top: it may reflect the animal's experience. If so, the neural activity would be similar if the animal goes through the same experience twice (left), analogous to the sequences on the timescale of seconds. In this case, the correlation between a pair of population vectors from different experiences will decay as the difference in their time, each within its experience, increases (right). Bottom: alternatively, the slow dynamics may be solely driven by the stochastic noise in neural systems and therefore drift randomly during different experiences (left). In this case, the correlations described above would not have any pattern (right). 
Exp 1: Treadmill running (Mau et al., 2018)

Exp 2: Spatial alternation (Levy et al., 2019)

Exp 3: Linear track (Rubin et al., 2015)

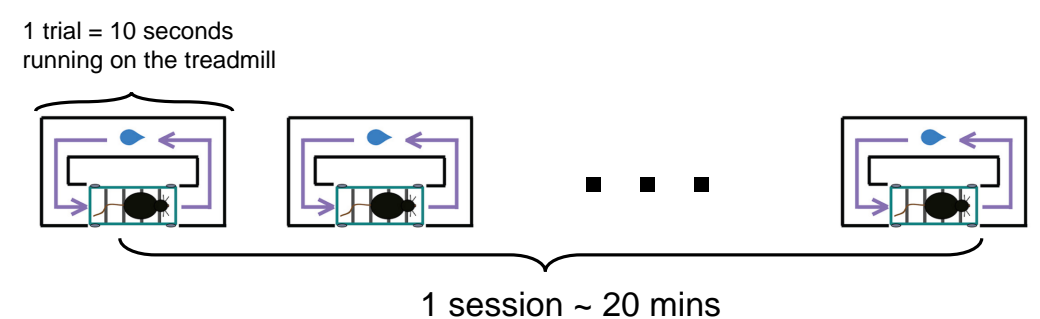

1 trial $=$ study phase (blue) + test phase (red)

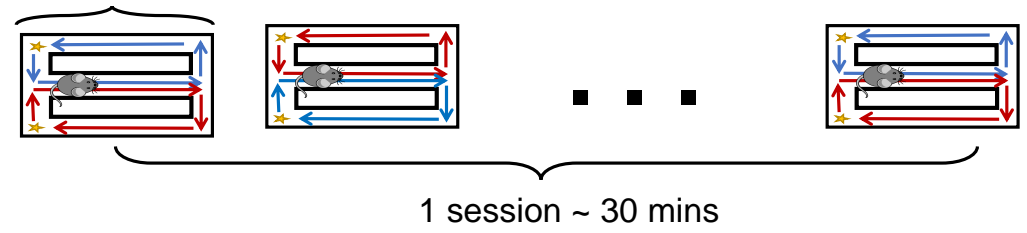

1 trial $=3$ minutes of running

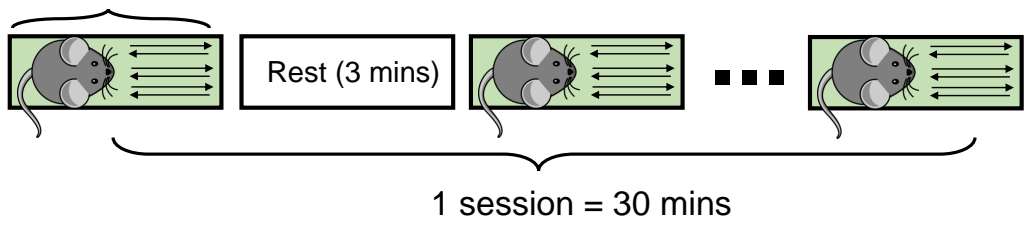

Figure 2. Two timescales in the structure of the experiments. For each experiment studied in this paper, the animals are trained to perform some task for several seconds-long trials in a recording session spanning tens of minutes. The calcium activity of the same neurons are recorded across sessions. During the treadmill running task (Experiment 1), mice are trained to run on the treadmill for 10 seconds before going to the opposite side of the maze to collect a water reward. The mice perform the same task for tens of trials each session for a total of around 20 minutes. For the spatial alternation task (Experiment 2), mice are trained to alternate between left and right turns in a T-maze to collect food rewards. Each trial consists of a study and test phase where mice have to turn to opposite directions at the choice point. Mice perform tens of trials for a total of around 30 minutes during each session. For the linear track experiment, mice are trained to run back and forth on a linear track to collect water rewards at both ends of the track. Each trial is about 3 minutes long and is separated by 3-minute resting periods where mice are placed in a separate box. Each session consists of 5 pairs of running and resting trials for a total of 30 minutes. See Methods section for more details of each experiment. 

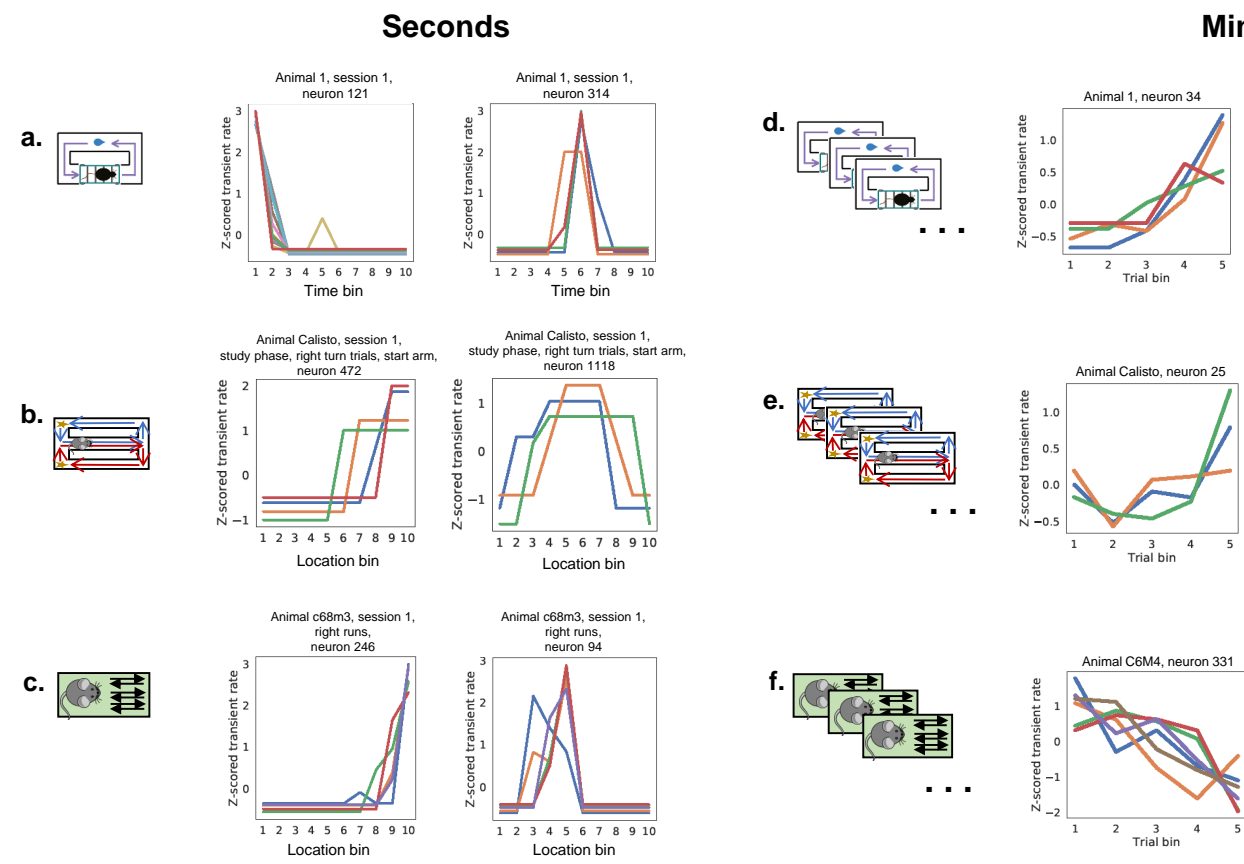

Minutes

a

Location bin e.
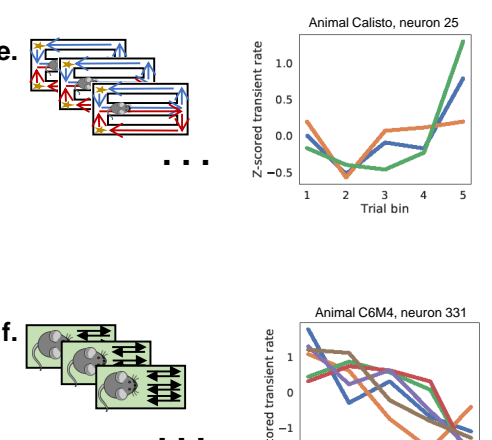

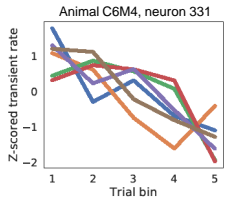

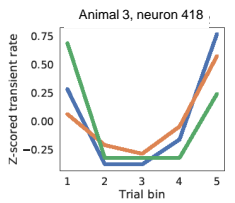

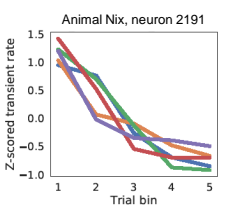

Figure 3. Many hippocampal neurons exhibit consistent dynamics across seconds and across minutes. a-c. Example neurons with consistent dynamics across trials for each of the experiments. In a, the 10-second running period is evenly divided into 10 time bins. In $\mathbf{b}$, the start arm of the maze is evenly divided into 10 location bins. In $\mathbf{c}$, the linear track is evenly divided into 10 location bins, and neural activity is averaged over all runs within a 3-minute trial. See Methods for details. d-f. Example neurons with consistent activity across sessions. Trials within each session are evenly divided into 5 trial bins. Each line represents the z-scored calcium transient rate over one trial/session. Inactive trials/sessions are not shown. See Supplementary figures S1 and S2 for more example neurons.

\section{Single hippocampal neurons have consistent activity across seconds and minutes}

We first plotted the normalized calcium transient density of individual Region of Interests (ROIs) against position or time within a trial. For each ROI we only included trials where it had at least one calcium transient event during the trial period examined. We observed that many ROIs have consistent activity within a trial (Figure 3a-c). For example, some ROIs always have higher activity at a particular time bin (Figure 3a, right) or location bin (Figure 3b-c, right) during each included trial. Other ROIs have higher activity at the start (Figure 3a, left) or end (Figure 3b-c, left) of each active trial. At longer time scales, similar consistent neural activity was observed when activity was plotted against trial number (Figure 3d-f, Methods). For example, some ROIs consistently increase (Figure 3d-e, left) or decrease (Figure 3f, left and 3e, right) their calcium activity across trials within a session. Other ROIs are consistently more active during particular trials within the session (Figure 3f, right).

To quantify the extent to which single neurons fire consistently across the population, we computed a firing consistency rank for each neuron, which is a number between zero and 
one that represents the consistency of that neuron's calcium dynamics between pairs of trials or sessions compared to chance (see Methods for details). We found that in all experiments and for both timescales, the distributions of the firing consistency rank are significantly skewed towards one compared to those obtained from the shuffled data (Figure 4). A Kolmogorov-Smirnov test between the distribution from true and shuffled data showed reliable differences in all cases, panel a $p<.001, n=1860$; panel b $p<.001, n=3525$; panel c $p<.001, n=1202$; panel d $p<.001, n=1330$, panel e $p<.001, n=4078$; panel $\mathrm{f}$ $p<.01, n=1673$. This indicates there are significantly more neurons that have consistent dynamics both across trials and across sessions than would be expected by chance.

There could be different types of single cell dynamics that contribute to the observed high firing consistency across repeated experiences. For example, some cells could have gradually increasing or decreasing activity, or they could exhibit non-monotonic dynamics over time such as those of time cells. To disentangle these two types of single cell dynamics, we used a similar method as above to construct a firing linearity rank for each neuron. The right panels of Figure 4 show the joint scatter plot of the firing consistency rank and the firing linearity rank for each of the experiments. As can be seen, there is a wide distribution of firing linearity across the population. This indicates that there is a diversity of consistent temporal dynamics both across a trial and a session. Firing linearity rank on the timescale of minutes and seconds are not significantly correlated with one another across neurons (Supplementary figure S14d-f).

\section{Hippocampal population dynamics are consistent both over seconds and min- utes}

The single cell analysis above left out trials or sessions where a given neuron is not active (does not have any calcium transient during the selected time period). To examine whether the consistency is present when the full ensemble of neurons are considered, we next investigated the consistency of the population-level dynamics across trials and sessions. To this end, we computed the cosine similarity between pairs of population vectors from different trials (Figure 5a-c) or sessions (Figure 5d-f) and assembled them into a matrix (see Methods for details). We found that in all experiments and across both timescales, the matrices exhibit a pattern where the values are highest along the diagonal, which indicates that the population dynamics are consistent across repeated trials and sessions (c.f., Figure 1b). Statistical significance was evaluated using a permutation test (see Methods for details). The degree to which all matrices show a diagonal pattern are greater than the heatmaps obtained from 10,000 random shuffles of the data. Notably, for the acrosstrial similarity matrix in the treadmill running task, the high-valued region spreads out later in the trial, indicating that the population dynamics slow down as time progresses (Figure 5a). This is consistent with the observation in the original study that the number density of sequentially-activated time cells goes down in time (Mau et al., 2018).

\section{Discussion}

In this paper, we show that the firing dynamics of hippocampal neurons are consistent over both seconds and minutes. The novel observation is that slow dynamics over minutes include slow sequences and are not simply random drifts (Figure 5d-f, Figure 1b). This 


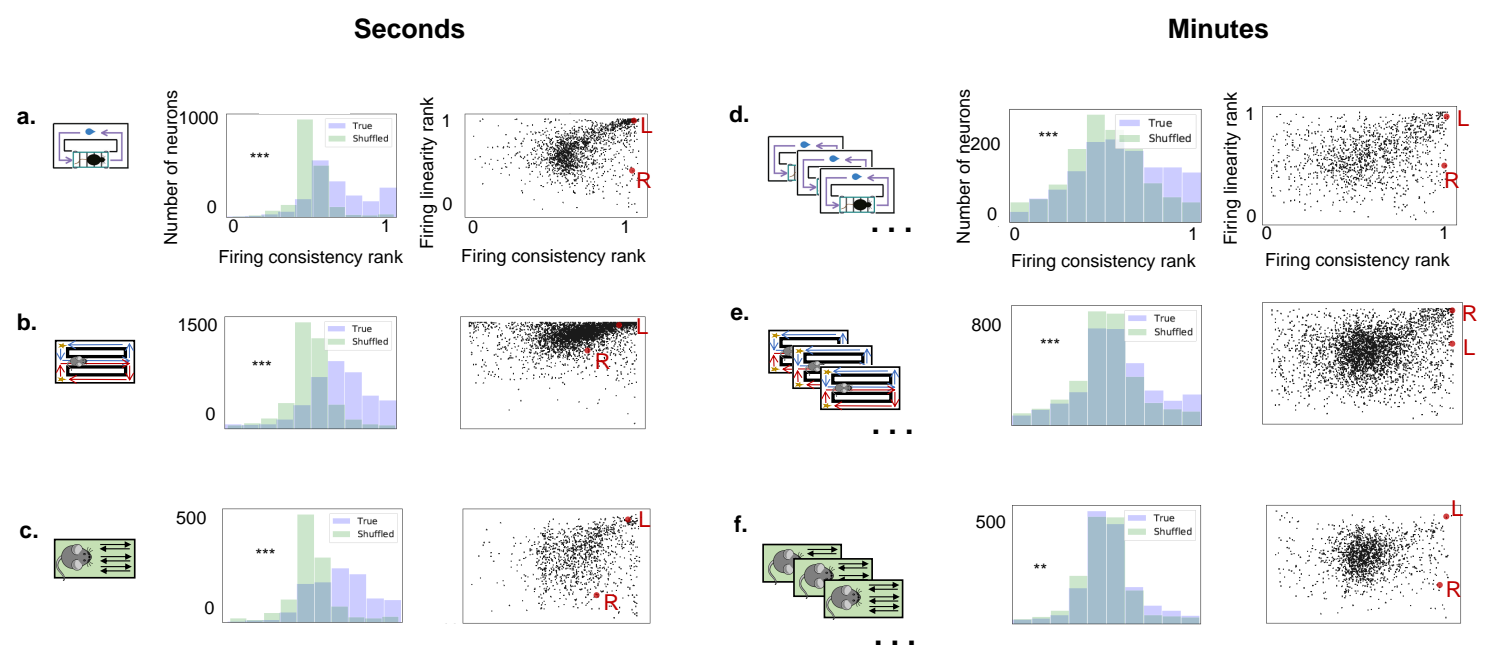

Figure 4. Many hippocampal neurons have consistent responses across seconds and across minutes. For each neuron, a firing consistency rank was computed to estimate how consistent the single cell dynamics are for within trial dynamics (a-c) and for across-trial dynamics (d-f). The histograms show the distribution of firing consistency rank relative to a surrogate distribution. To the extent these distributions differ, one can conclude that there are consistent sequences. To evaluate the degree to which dynamics were simply monotonic changes in firing rate, we also computed a firing linearity rank. The scatter plots show the consistency rank and linearity rank for each neuron. Red dots indicate the example single neurons shown in Figure 3. L and R refer to the example neuron on the left and right of each panel in Figure 3, respectively. See Methods for more details. Supplementary figures S3-S13 show the same analyses for each trial type, session and animal.

population effect results from a significant proportion of neurons with consistent dynamics over repeated experiences (Figure 4). These neurons have both monotonic and more complex activity modulations across each experience (Figures 3 and 4 and Supplementary figures S1 and S2). Therefore, the hippocampal neurons exhibit consistent dynamics over two nested timescales - changing both systematically within a trial and systematically within a session - in each of these experiments.

This result suggests that the spatiotemporal context as represented by population of neurons in the hippocampus has meaningful dynamics over multiple timescales, from seconds to minutes. The sensitivity to multiple timescales may enable the hippocampus to adaptively encode natural stimuli, which vary at many different scales (Voss \& Clarke, 1975; Hasson, Yang, Vallines, Heeger, \& Rubin, 2008) and account for the self-similar structure in hippocampal correlations (Meshulam, Gauthier, Brody, Tank, \& Bialek, 2019). The responsiveness of hippocampal dynamics provides a constraint for behavioral models of human memory. Models that rely on boundaries and event segmentation (Farrell, 2012; Franklin, Norman, Ranganath, Zacks, \& Gershman, 2020) must be able to construct and utilize segments over multiple nested scales. Similarly, neural models for sequence generation (Buzsáki \& Tingley, 2018; Rajan, Harvey, \& Tank, 2016; Howard et al., 2014) must 


\section{Representations in timescales of seconds}

a.

b.

c.

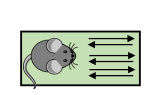

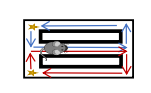
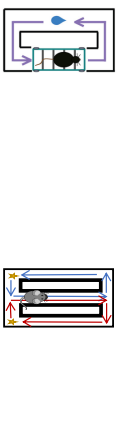

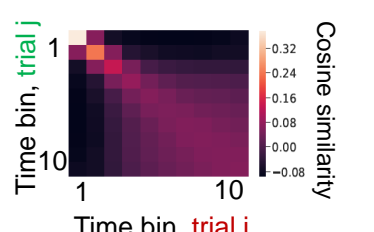

Time bin, trial i

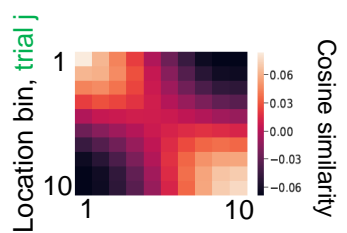

Location bin, trial i

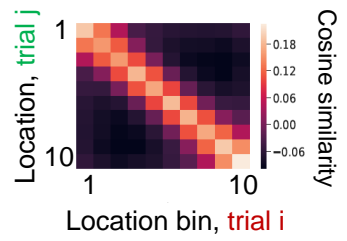

d.

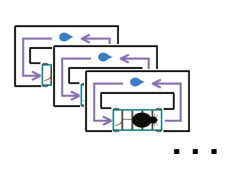

Representations in timescales of minutes e.

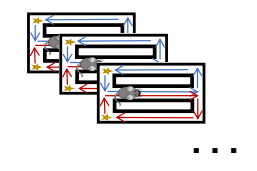

f.

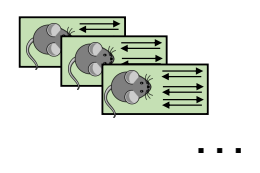

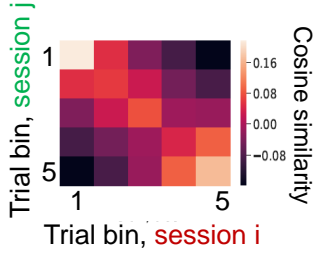
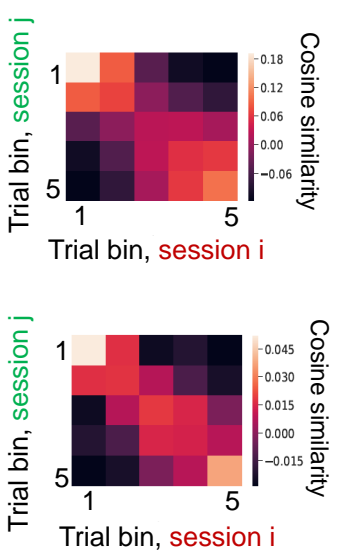

Figure 5. Population dynamics in the hippocampus are consistent across both seconds and minutes. a-c. Consistent population dynamics within seconds-long trials. Each element of the matrix is the cosine similarity between a pair of population vectors at different binned locations within a trial, average over pairs of trials, trial types (for $\mathrm{b}$ and $\mathrm{c}$ ), sessions and animals. Critically, the population vectors are taken from the same time or location bin, but from different trials. See Methods for the details of how binning was performed for the different tasks. All three experiments (a: treadmill task, b: spatial alternation, c: linear track) show higher correlation along the diagonal, indicating that the population goes through a consistent sequence within each trial. This is as we would expect from the known properties of time cells (a) and place cells (b and $\mathbf{c}$ ). d-f. Consistent population dynamics across trials. Similar to a-c, except each element of the matrix is the cosine similarity between a pair of population vectors from two different sessions, averaged over all pairs of sessions and animals. Rather than computing population vectors from bins of time or space within a trial, population vectors were computed by averaging over entire trials (see Methods for details). The similarity between population vectors from different recording session was then computed for different pairs of trial bins. The elements of all three matrices are highest on the diagonal and gradually decrease off-diagonal, similar to the matrices over a trial (a-c). This indicates that the population dynamics are also consistent across minute-long sessions. 
have the capacity to generate sequences at many different scales.

\section{Possible causes of slow dynamics}

Possible non-physiological causes. There are recording artifacts specific to calcium imaging that could conceivably cause slow dynamics. For example, photobleaching could cause the calcium fluorescence signal to decrease gradually during each imaging session or gradual heating of the brain could potentially produce stereotypical changes in the apparent calcium fluorescence signal for each ROI over the course of an imaging session. It is difficult to reconcile these artifactual accounts of slow dynamics with non-monotonic patterns of firing over the session or the striking similarity between effects observed across trials to the effects within trial. The findings within-trial are quite consistent with results using extracellular electrodes. We conclude that it is likely that the slow dynamics are physiological in origin.

Variables correlated with time during a session. It is possible that the slow dynamics observed in the hippocampus are not due to memory per se but reflect consistent slow dynamics in the environment or internal state of the animal over the course of the recording session. There are several possibilities for such variables. For example, the satiety of the animal presumably decreases over the course of each recording session. Indeed, it has recently been reported that thirst level has a dramatic impact on the population activity in multiple brain regions in mice over the course of minutes (Allen et al., 2019). Slow drifts over minutes in area V4 and prefrontal cortex of monkey are correlated with systematic changes in animal's behavior during a perceptual decision making task (Cowley et al., 2020). Microdialysis of acetylcholine shows higher levels of acetylcholine when an animal is first removed from the home cage and placed in a task (Acquas, Wilson, \& Fibiger, 1996). Acetelcholine levels decrease over time on the scale of minutes and have been shown to depolarize hippocampal neurons (Cole \& Nicoll, 1984) and increase firing rate (Fu et al., 2014). In all of these cases, the sequential activation of cells over the recording session would require that the hippocampus codes for a monotonically changing variable with a sequence of receptive fields. Indeed, this kind of pattern has been observed for hippocampal receptive fields as a function of smooth changes in frequency of a behaviorally-relevant tone (Aronov, Nevers, \& Tank, 2017).

In some sense the empirical story for very slow sequences is analogous to the empirical story for place cells or time cells shortly after their initial report. Although a consensus has emerged that place cells and time cells express spatial and temporal relationships between events in the service of memory (e.g., Eichenbaum, 2017), this view only emerged after extensive empirical studies. For instance, a neuron that fires when the animal is in a specific position of an environment could be responding to the visual stimuli available at that location, the particular configuration of auditory stimuli available, or olfactory cues present on the track. Early studies ruled out a series of possible confounds of spatial position (e.g., Quirk, Muller, \& Kubie, 1990; Save, Cressant, Thinus-Blanc, \& Poucet, 1998). Similarly, it is possible that initial reports of time cells could have been solely a reaction to a behavioral confound during the delay such as a stereotyped behavior. However, time cells have been observed in head-fixed animals and different stimuli trigger distinct sequences (e.g., Pastalkova et al., 2008; Taxidis et al., 2020; Cruzado, Tiganj, Brincat, Miller, \& Howard, 2020), ruling out most possible confounds. 
Slow dynamics as memory for the past. It has been clearly established that hippocampal time cells express memory for the time and identity of past events (e.g., Pastalkova et al., 2008; Taxidis et al., 2020; Cruzado et al., 2020). The most interesting possible cause of the slow dynamics observed here is that they reflect the same computational mechanism, but over much slower time scales than within-trial time cells. This raises the question of how the neurons in the hippocampus could generate such slow sequences. One possibility is that the slowness of the hippocampal representation is inherited from its inputs. Tsao et al. (2018) observed very slow changes in the firing of neurons in lateral entorhinal cortex (LEC). In that study, LEC neurons changed their firing rate abruptly and then relaxed back to baseline with a broad range of decay rates. For instance, upon entry to a particular environment, a neuron might rapidly increase its firing rate and then decay exponentially back to baseline over several minutes. Other neurons ramped over the entire recording session so there was a variety of decay rates across neurons. This slowly-varying signal in LEC at the scale of minutes could be a cause of the slow sequences we observed in hippocampus. The same mechanism could also cause time cells on the scale of seconds. Bright et al. (2020) studied neurons in monkey EC during a visual task. After an image was presented, the neurons changed firing rate then gradually relaxed back to baseline with a variety of decay rates. Because there was a variety of relaxation rates it was possible to decode time since image onset over several seconds (see also Hyde \& Strowbridge, 2012). Very long-lasting firing in EC has been observed in vitro (Egorov, Hamam, Fransén, Hasselmo, \& Alonso, 2002) and is believed to be caused by the nonspecific calcium-sensitive (CAN) cationic current. Computational models show that the CAN current can also induce slowly decaying firing with a variety of decay rates in a simple integrate-and-fire neuron model (Tiganj, Hasselmo, \& Howard, 2015). Computational models have shown that the temporal information carried by slowly-decaying activity can be used to generate a population of sequentially-activated time cells (Shankar \& Howard, 2012; Howard et al., 2014; Liu, Tiganj, Hasselmo, \& Howard, 2019; Rolls \& Mills, 2019).

In this study we showed that slow hippocampal sequences are consistent across trials in a recording session. The definitive test of whether hippocampal sequences reflect a very slow form of memory is whether the identity of events on previous trials can be decoded. In these studies, the trials were very similar to one another. Studies of in learning tasks have shown robust memory for information about the identity of past trials. For instance, neural activity on one trial of a learning task can be used to decode the identity and reward history of previous trials in orbitofrontal cortex and piriform cortex of rats (Schoenbaum \& Eichenbaum, 1995b, 1995a), posterior parietal cortex of mice (Morcos \& Harvey, 2016) and anterior cingulate cortex, dorsolateral prefrontal cortex and lateral intraparietal cortex of monkeys (Bernacchia, Seo, Lee, \& Wang, 2011).

A recent study shows that there are neurons in the hippocampus CA1 of mice that encode the number of laps that the animal has traversed in a task where they have to run four consecutive laps to obtain a reward (Sun, Yang, Martin, \& Tonegawa, 2020). Some of the neurons reported in our work indeed show elevated activity at a particular trial bin, but we have also observed neurons whose activity exhibit more complex modulations by trial bin number (Figure 3d-f and Supplementary figures S1- S2). It remains to be shown the exact functions of the slow sequences reported here. 


\section{Methods}

\section{Behavioral tasks and calcium imaging}

The treadmill running task. Four mice were trained to traverse a rectangular track followed by running in place on a motorized treadmill for $10 \mathrm{~s}$ at a constant velocity to receive sucrose water reward after traversing an additional part of the track (Figure 2, Experiment 1). During each session, the mice completed between 35-37 trials. A total of 4 sessions were performed for each mouse.

Mice received infusions of AAV9- Syn-GCaMP6f (U Penn Vector Core). Imaging data in dorsal CA1 were acquired using a commercially available miniaturized head-mounted epifluorescence microscope (Inscopix). The raw video was pre-processed using an image segmentation algorithm called Tenaspis (D.W. Sullivan et al., 2017, Soc. Neurosci., abstract, software available at https://github.com/SharpWave/TENASPIS) to extract ROIs and assign calcium transient events to each ROI. This algorithm is designed to better distinguish between overlapping ROIs. The calcium transients it detects correspond to the rising phase of the calcium fluorescence. 296-1136 ROIs were identified during each session.

In order to identify the same neurons across recording sessions that are days apart, ROIs were cross-registered across days. Briefly, this was done by first aligning the field of view of each session to the first session using vasculature as stationary landmarks via image registration software from MATLAB's Image Processing Toolbox, assuming rigid geometric transformation. Then, cells were successively registered from each session to the next session (Day 1 to Day 2, Day 2 to Day 3, etc.). Cells were registered by searching for the nearest ROI with a threshold that the ROI centroids must be within 3.3 microns apart. To ensure that neurons do not drift excessively across days, the first day's neurons were registered with the last day's neurons, and any registrations between Day 4 and Day 1 that are different from Day 4 and Day 3 were discarded.

More details about the behavioral setup and the calcium imaging experiment can be found in the Methods section of Mau et al. (2018).

The spatial alternation task. Four mice were trained on a spatial alternation task, during which they alternated between "study" and "test" trials. On study trials, mice were placed on the center stem of maze, ran to the crossroads, where a removable barrier forced them to run down one of the two return arms and received a reward of chocolate sprinkle. They were then moved into the delay area located at the bottom of the center stem, waited through a 20-second delay, and the delay barrier was lifted to start the test trial. On a test trial, mice again ran up the center stem to the crossroads but this time there was no barrier and they had to remember the direction they traveled on the study trial and turn to the return arm opposite to the preceding study trial in order to receive a reward. They then moved to the delay area, and were placed in their home cage to wait through a 15-25 second inter-trial interval while the next study trial was set up (Figure 2, Experiment 2). Mice completed between 25 and 40 study-test trial pairs per session. Each of these trial pairs is considered a "trial" in the analysis in the main text.

The experimental procedures for calcium imaging and the data pre-processing pipeline are the same as the treadmill running task. 1149-3165 ROIs were identified for each session. The cell cross-registration procedure is slightly different from the treadmill running task. 
Sessions were aligned to a "base" session from the middle of the recording schedule using 25-40 "anchor" cells. Cells with centers within 3 microns were identified as the same cell. More details about the experimental setup can be found in Levy et al. (2019).

The linear track task. Three mice (2 were injected with AAV2/5-CaMKIIa-GCaMP6f and one was a Thy1-GCaMP6f transgenic; Jackson stock number 025393) were trained to run back and forth on an elevated $96 \mathrm{~cm}$ long linear track. They received water sweetened with lemon flavored fruit juice concentrate at each end of the track. An overhead camera (DFK 33G445, The Imaging Source, Germany) was used to record mouse behavior. Each session consisted of five 3-min trials with 3-min intertrial intervals. 7-8 sessions were performed in total for each mouse. Sessions were performed in the morning and the afternoon in alternation.

An integrated miniature fluorescence microscope (nVistaHD, Inscopix) was used to obtain the imaging data from the CA1 region of the hippocampus. Imaging data was preprocessed using commercial software (Mosaic, Inscopix) and custom MATLAB routines as previously described in Ziv et al., 2013. Spatial filters corresponding to individual ROIs were first identified using a cell-sorting algorithm that utilizes principal component analysis and independent component analysis (PCA and ICA, Mukamel, Nimmerjahn, \& Schnitzer, 2009) and then subjected to further manual cell sorting (see the "Materials and methods" section of Rubin et al. (2015) for more details). Calcium transient events were identified when the amplitude of the calcium traces $\left(\frac{d F}{F}\right)$ crossed a threshold of 5 median absolute deviations (MAD). Further measures were taken to avoid the detection of multiple peaks as well as the spillover of the calcium fluorescence to neighboring cells (see the "Materials and methods" section of Rubin et al. (2015) for more details).

Registration of cells across sessions was performed by first aligning the field of view in each session to the first session and then computing the spatial correlation between ROI centroids in the reference coordinate system. Pairs with spatial correlation $>0.7$ or distance $<5 \mu \mathrm{m}$ were registered as the same neuron. For the full detail on the experimental setup please refer to the "Materials and methods" section of Rubin et al., 2015.

\section{Data analysis methods}

\section{Coarse-graining of calcium activity.}

Coarse-graining for the across session dynamics. To extract the slow neuronal dynamics across multiple trials while filtering out the fast within-trial dynamics, the neural activity was first temporally coarse-grained before further analysis. When comparing a pair of sessions, the session with more trials was first truncated to have the same number of trials as the other session. Then, all the remaining trials within a session were evenly divided into 5 trial bins by using the array_split function in Numpy. Then the neural activity for each ROI is the calcium transient density over that trial bin. Therefore, the temporally coarse-grained activity of an ROI $n$ during a session $i$ was represented by a time series with length 5: $v_{n, i}=\left\{v_{n, i, 1}, v_{n, i, 2}, v_{n, i, 3}, v_{n, i, 4}, v_{n, i, 5}\right\}$. Furthermore, since we are interested in the temporal modulation of the neural activity rather than the absolute magnitude of the activity, the coarsed-grained activity of each cell was z-scored across trial bins. We chose 5 as the number of time bins within a session since each session in the linear track task 
consists of 5 running trials (Figure 2, Experiment 3), and we wish to keep the way trials are divided consistent across experiments. Similar results were obtained for the treadmill running task and the spatial alternation task by using 10 trial bins. Furthermore, we only averaged over the calcium activity during time periods when the animal's behavior is under experimental control. In the treadmill running task, the time periods used are when the animal is running on the treadmill for 10 seconds. In the spatial alternation experiment, the time periods used are when the animal is running along the start arm. In the linear track experiment, the time periods used are when the animal's position is within the middle $60 \%$ of the linear track.

Coarse-graining for the within-trial dynamics.. For the across-trial analysis, coarse-graining was done in a similar way by computing the calcium transient density over 10 time bins or location bins within each trial. For the treadmill running task, calcium event rate was averaged over each second during the 10-second running period. For the spatial alternation task, the start arm was evenly divided into 10 location bins and total number of calcium transient events within each bin divided by the amount of time the animal spent in that bin was computed. Unless otherwise specified, all analysis was performed separately for the two task epochs (study and test) and two trial types (turn left and turn right) and the results were averaged. For the linear track task, the within-trial neural activity was computed by first computing for each individual run the number of calcium transient events within each location bin divided by the amount of time the animal spent in that bin, and then averaging this quantity over all runs within a 5-minute trial. This was done for the two running directions separately, and the results were averaged. The 10 location bins span the middle $30 \%$ of the track. We chose the middle $30 \%$ of the linear track because this is similar to the length of the start arm in the spatial alternation task. Lastly, for all experiments, the activity of each neuron was z-scored across all spatial or time bins for each trial.

Cosine similarity between population vectors. For Figure $5 \mathrm{~d}-\mathrm{f}$, we computed the cosine similarity between pairs of population activity vectors during different sessions after they were coarse-grained and z-scored as described above. We then built a matrix where each element represents the cosine similarity between a pair of population vectors at two trial bins during two different sessions, averaged over all pairs of sessions and all animals.

For Figure 5a-c, population vectors were computed by averaging neural activity over time or location bins within each trial, as described above. Then a similar correlation matrix was constructed where each element is the cosine similarity between a pair of population vectors from different trials.

To test that the matrix shows a significant diagonal pattern, neural activity across all bins within each session (Figure 5d-f) or trial (Figure 5a-c) was shuffled 10000 times independently for each neuron and matrices from this shuffled data were constructed. We characterized the degree to which each matrix shows a diagonal pattern by an index $d$, which equals the difference between the average value of the near-diagonal matrix elements to that of the off-diagonal matrix elements. The near-diagonal matrix elements are those whose row and column indices are differed by less than half the dimension of the matrix. Mathematically, $d=\left\langle M_{i j}\right\rangle_{|i-j|<\frac{N}{2}}-\left\langle M_{i j}\right\rangle_{|i-j| \geq \frac{N}{2}}$, where $N$ is the dimension of the matrix. Then we counted how many matrices constructed from the shuffled data have a value $d$ greater than the matrix obtained from the true data. As a result, none of the 10000 
matrices from the shuffled data has a higher $d$ than the matrices in Figure 5.

Firing consistency rank. To assess the consistency of the single neuron dynamics across repeated trials or sessions, we computed a firing consistency rank for each neuron. For each cell $n$ and each pair of sessions or trials (for example $i$ and $j$ ), we computed the Pearson's correlation coefficient between the coarse-grained activity vectors $v_{n, i}$ and $v_{n, j}$ obtained from the method described above. Then we shuffled the entries in each activity vector and computed the Pearson's correlation coefficient again. This was repeated for 100 times, and all the Pearson's correlations were ranked from the lowest to the highest. We then found the percentile where the true Pearson's correlation is at among all the shuffles (if there are multiple shuffles that yield the same Pearson's correlation as the true data, the median percentile was used). Finally, the firing consistency rank is the mean percentile across all pairs of sessions (or trials). The rank across trials (Figure 4a-c) was further averaged across trial types (for Figure 4a, c) and sessions. Sessions or trials where the neuron does not have any calcium transient event during the selected time period were excluded from the analysis.

Firing linearity rank. To disentangle the gradually ramping/decaying activity from more complex temporal modulations, we computed a firing linearity rank for each neuron. For a given neuron $n$ and session (or trial) $i$, we fitted a linear model as a function of the bin number for the coarse-grained activity $v_{n, i}$ of that neuron. The F-statistic of this linear model was computed along with those obtained from 100 shuffled activity vectors (shuffling was performed in the same way as in computing the firing consistency rank). The percentile of the true F-statistic among all the shuffles was computed (if the F statistic of multiple shuffles equal the true $\mathrm{F}$ statistic, the median percentile was used). This percentile was then averaged across all sessions (or trials) to obtain the firing linearity rank for that neuron. The rank across trials (Figure 4a-c) were further averaged across trial types (for Figure 4a, c) and sessions. For each neuron, sessions (or trials) where no calcium transients were observed were excluded from the analysis.

\section{References}

Acquas, E., Wilson, C., \& Fibiger, H. C. (1996). Conditioned and unconditioned stimuli increase frontal cortical and hippocampal acetylcholine release: effects of novelty, habituation, and fear. Journal of Neuroscience, 16(9), 3089-3096.

Allen, W. E., Chen, M. Z., Pichamoorthy, N., Tien, R. H., Pachitariu, M., Luo, L., \& Deisseroth, K. (2019). Thirst regulates motivated behavior through modulation of brainwide neural population dynamics. Science, 364(6437).

Aronov, D., Nevers, R., \& Tank, D. W. (2017, Mar). Mapping of a non-spatial dimension by the hippocampal-entorhinal circuit. Nature, 543(7647), 719-722. doi: 10.1038/nature21692

Bernacchia, A., Seo, H., Lee, D., \& Wang, X. J. (2011). A reservoir of time constants for memory traces in cortical neurons. Nature Neuroscience, 14(3), 366-72.

Bright, I. M., Meister, M. L., Cruzado, N. A., Tiganj, Z., Buffalo, E. A., \& Howard, M. W. (2020). A temporal record of the past with a spectrum of time constants in the monkey entorhinal cortex. Proceedings of the National Academy of Sciences.

Buzsáki, G., \& Tingley, D. (2018). Space and time: The hippocampus as a sequence generator. Trends in cognitive sciences, 22(10), 853-869. 
Cai, D. J., Aharoni, D., Shuman, T., Shobe, J., Biane, J., Song, W., ... Silva, A. (2016). A shared neural ensemble links distinct contextual memories encoded close in time. Nature, 534(7605), 115-118.

Cole, A. E., \& Nicoll, R. (1984). Characterization of a slow cholinergic post-synaptic potential recorded in vitro from rat hippocampal pyramidal cells. The Journal of physiology, 352(1), 173-188.

Cowley, B. R., Snyder, A. C., Acar, K., Williamson, R. C., Byron, M. Y., \& Smith, M. A. (2020). Slow drift of neural activity as a signature of impulsivity in macaque visual and prefrontal cortex. bioRxiv.

Cruzado, N. A., Tiganj, Z., Brincat, S. L., Miller, E. K., \& Howard, M. W. (2020). Conjunctive representation of what and when in monkey hippocampus and lateral prefrontal cortex during an associative memory task. Hippocampus, 30(12), 1332-1346. Retrieved from https://onlinelibrary.wiley.com/doi/abs/10.1002/hipo.23282 doi: https://doi.org/10.1002/hipo.23282

DuBrow, S., Rouhani, N., Niv, Y., \& Norman, K. A. (2017). Does mental context drift or shift? Current opinion in behavioral sciences, 17, 141-146.

Egorov, A. V., Hamam, B. N., Fransén, E., Hasselmo, M. E., \& Alonso, A. A. (2002). Graded persistent activity in entorhinal cortex neurons. Nature, 420 (6912), 173-8.

Eichenbaum, H. (2017). On the integration of space, time, and memory. Neuron, 95(5), 1007-1018.

Ekstrom, A. D., \& Ranganath, C. (2018). Space, time, and episodic memory: The hippocampus is all over the cognitive map. Hippocampus, 28(9), 680-687.

Ezzyat, Y., \& Davachi, L. (2014). Similarity breeds proximity: Pattern similarity within and across contexts is related to later mnemonic judgments of temporal proximity. Neuron, 81 (5), 1179-1189.

Farrell, S. (2012). Temporal clustering and sequencing in short-term memory and episodic memory. Psychological Review, 119(2), 223-71. doi: 10.1037/a0027371

Franklin, N. T., Norman, K. A., Ranganath, C., Zacks, J. M., \& Gershman, S. J. (2020). Structured event memory: A neuro-symbolic model of event cognition. Psychological Review, $127(3), 327$.

Fu, Y., Tucciarone, J. M., Espinosa, J. S., Sheng, N., Darcy, D. P., Nicoll, R. A., .. Stryker, M. P. (2014). A cortical circuit for gain control by behavioral state. Cell, 156(6), 1139-1152.

Hasselmo, M. E. (2012). How we remember: Brain mechanisms of episodic memory. Cambridge, MA: MIT Press.

Hasson, U., Yang, E., Vallines, I., Heeger, D. J., \& Rubin, N. (2008). A hierarchy of temporal receptive windows in human cortex. Journal of Neuroscience, 28(10), 2539-50. doi: 10.1523/JNEUROSCI.5487-07.2008

Healey, M. K., Long, N. M., \& Kahana, M. J. (2019). Contiguity in episodic memory. Psychonomic bulletin \& review, 26(3), 699-720.

Howard, M. W., Fotedar, M. S., Datey, A. V., \& Hasselmo, M. E. (2005). The temporal context model in spatial navigation and relational learning: Toward a common explanation of medial temporal lobe function across domains. Psychological Review, 112(1), 75-116.

Howard, M. W., MacDonald, C. J., Tiganj, Z., Shankar, K. H., Du, Q., Hasselmo, M. E., 
\& Eichenbaum, H. (2014). A unified mathematical framework for coding time, space, and sequences in the hippocampal region. Journal of Neuroscience, 34(13), 4692-707. doi: 10.1523/JNEUROSCI.5808-12.2014

Howard, M. W., Youker, T. E., \& Venkatadass, V. (2008). The persistence of memory: Contiguity effects across several minutes. Psychonomic Bulletin \& Review, 15(PMC2493616), 58-63.

Hsieh, L.-T., Gruber, M. J., Jenkins, L. J., \& Ranganath, C. (2014). Hippocampal activity patterns carry information about objects in temporal context. Neuron, 81(5), 11651178.

Hyde, R. A., \& Strowbridge, B. W. (2012). Mnemonic representations of transient stimuli and temporal sequences in the rodent hippocampus in vitro. Nature Neuroscience, 15(10), 1430-8. doi: 10.1038/nn.3208

Kahana, M. J. (1996). Associative retrieval processes in free recall. Memory $\&$ Cognition, 24, 103-109.

Kraus, B. J., Robinson, R. J., 2nd, White, J. A., Eichenbaum, H., \& Hasselmo, M. E. (2013). Hippocampal "time cells": time versus path integration. Neuron, 78(6), 1090-101. doi: 10.1016/j.neuron.2013.04.015

Levy, S. J., Kinsky, N. R., Mau, W., Sullivan, D. W., \& Hasselmo, M. E. (2019). Hippocampal spatial memory representations in mice are heterogeneously stable. bioRxiv, 843037.

Liu, Y., Tiganj, Z., Hasselmo, M. E., \& Howard, M. W. (2019). A neural microcircuit model for a scalable scale-invariant representation of time. Hippocampus, 29 (3), 260-274.

MacDonald, C. J., Lepage, K., Eden, U., \& Eichenbaum, H. (2010). Hippocampal neurons encode the temporal organization of non-spatial event sequences. Society for Neuroscience Abstracts, 36, 100.15.

MacDonald, C. J., Lepage, K. Q., Eden, U. T., \& Eichenbaum, H. (2011). Hippocampal âĂIJtime cellsâĂİ bridge the gap in memory for discontiguous events. Neuron, 71 (4), $737-749$.

Mankin, E. A., Diehl, G. W., Sparks, F. T., Leutgeb, S., \& Leutgeb, J. K. (2015). Hippocampal CA2 activity patterns change over time to a larger extent than between spatial contexts. Neuron, 85(1), 190-201. doi: 10.1016/j.neuron.2014.12.001

Mankin, E. A., Sparks, F. T., Slayyeh, B., Sutherland, R. J., Leutgeb, S., \& Leutgeb, J. K. (2012). Neuronal code for extended time in the hippocampus. Proceedings of the National Academy of Sciences, 109, 19462-7. doi: 10.1073/pnas.1214107109

Manns, J. R., Howard, M. W., \& Eichenbaum, H. B. (2007). Gradual changes in hippocampal activity support remembering the order of events. Neuron, 56(3), 530-540.

Mau, W., Sullivan, D. W., Kinsky, N. R., Hasselmo, M. E., Howard, M. W., \& Eichenbaum, H. (2018). The same hippocampal cal population simultaneously codes temporal information over multiple timescales. Current Biology, 28(10), 1499-1508.

Meshulam, L., Gauthier, J. L., Brody, C. D., Tank, D. W., \& Bialek, W. (2019). Coarse graining, fixed points, and scaling in a large population of neurons. Physical review letters, 123(17), 178103.

Miller, J. F., Lazarus, E. M., Polyn, S. M., \& Kahana, M. J. (2013). Spatial clustering during memory search. Journal Experimental Psychology: Learning, Memory and Cogntion, 39(3), 773-81. doi: 10.1037/a0029684 
Morcos, A. S., \& Harvey, C. D. (2016). History-dependent variability in population dynamics during evidence accumulation in cortex. Nature neuroscience, 19(12), 1672-1681.

Moser, E. I., Kropff, E., \& Moser, M.-B. (2008). Place cells, grid cells, and the brain's spatial representation system. Annual Review of Neuroscience, 31, 69-89. doi: 10.1146/annurev.neuro.31.061307.090723

Mukamel, E. A., Nimmerjahn, A., \& Schnitzer, M. J. (2009). Automated analysis of cellular signals from large-scale calcium imaging data. Neuron, 63(6), 747-60. doi: 10.1016/j.neuron.2009.08.009

O'Keefe, J., \& Dostrovsky, J. (1971). The hippocampus as a spatial map. preliminary evidence from unit activity in the freely-moving rat. Brain Research, 34(1), 171-175.

O'Keefe, J., \& Nadel, L. (1978). The hippocampus as a cognitive map. New York: Oxford University Press.

Pastalkova, E., Itskov, V., Amarasingham, A., \& Buzsaki, G. (2008). Internally generated cell assembly sequences in the rat hippocampus. Science, 321(5894), 1322-7.

Polyn, S. M., \& Kahana, M. J. (2008). Memory search and the neural representation of context. Trends in Cognitive Science, 12(1), 24-30.

Quirk, G. J., Muller, R. U., \& Kubie, J. L. (1990). The firing of hippocampal place cells in the dark depends on the rat's recent experience. Journal of Neuroscience, 10(6), 2008-17.

Rajan, K., Harvey, C. D., \& Tank, D. W. (2016). Recurrent Network Models of Sequence Generation and Memory. Neuron, 90(1), 128142. Retrieved from http://dx.doi.org/10.1016/j.neuron.2016.02.009 doi: 10.1016/j.neuron.2016.02.009

Rolls, E. T., \& Mills, P. (2019). The generation of time in the hippocampal memory system. Cell reports, 28(7), 1649-1658.

Rubin, A., Geva, N., Sheintuch, L., \& Ziv, Y. (2015). Hippocampal ensemble dynamics timestamp events in long-term memory. eLife, 4, e12247.

Save, E., Cressant, A., Thinus-Blanc, C., \& Poucet, B. (1998). Spatial firing of hippocampal place cells in blind rats. Journal of Neuroscience, 18(5), 1818-1826.

Schoenbaum, G., \& Eichenbaum, H. (1995a). Information coding in the rodent prefrontal cortex. II. Ensemble activity in orbitofrontal cortex. Journal of Neurophysiology, $74(2), 751-62$.

Schoenbaum, G., \& Eichenbaum, H. (1995b). Information coding in the rodent prefrontal cortex. I. Single-neuron activity in orbitofrontal cortex compared with that in pyriform cortex. Journal of Neurophysiology, 74 (2), 733-50.

Scoville, W. B., \& Milner, B. (1957). Loss of recent memory after bilateral hippocampal lesions. Journal of Neurology, Neurosurgery, and Psychiatry, 20, 11-21.

Shankar, K. H., \& Howard, M. W. (2012). A scale-invariant internal representation of time. Neural Computation, 24(1), 134-193.

Staresina, B. P., \& Davachi, L. (2009). Mind the gap: binding experiences across space and time in the human hippocampus. Neuron, 63(2), 267-76.

Sun, C., Yang, W., Martin, J., \& Tonegawa, S. (2020). Hippocampal neurons represent events as transferable units of experience. Nature Neuroscience, 23(5), 651-663.

Taxidis, J., Pnevmatikakis, E. A., Dorian, C. C., Mylavarapu, A. L., Arora, J. S., Samadian, K. D., .. Golshani, P. (2020). Differential emergence and stability of sensory and 
temporal representations in context-specific hippocampal sequences. Neuron.

Tiganj, Z., Hasselmo, M. E., \& Howard, M. W. (2015). A simple biophysically plausible model for long time constants in single neurons. Hippocampus, 25 (1), 27-37.

Tsao, A., Sugar, J., Lu, L., Wang, C., Knierim, J. J., Moser, M.-B., \& Moser, E. I. (2018). Integrating time from experience in the lateral entorhinal cortex. Nature, 561(7721), 57.

Tulving, E. (1983). Elements of episodic memory. New York: Oxford.

Uitvlugt, M. G., \& Healey, M. K. (2019). Temporal proximity links unrelated news events in memory. Psychological science, 30(1), 92-104.

Unsworth, N. (2008). Exploring the retrieval dynamics of delayed and final free recall: Further evidence for temporal-contextual search. Journal of Memory and Language, 59, 223-236.

Voss, R. F., \& Clarke, J. (1975). 1/f noise in music and speech. Nature, 258, 317-318.

Wallenstein, G. V., Eichenbaum, H. B., \& Hasselmo, M. E. (1998). The hippocampus as an associator of discontiguous events. Trends in Neuroscience, 21, 317-323.

Yonelinas, A. P., Ranganath, C., Ekstrom, A. D., \& Wiltgen, B. J. (2019). A contextual binding theory of episodic memory: systems consolidation reconsidered. Nature Reviews Neuroscience, 20(6), 364-375.

Ziv, Y., Burns, L. D., Cocker, E. D., Hamel, E. O., Ghosh, K. K., Kitch, L. J., .. Schnitzer, M. J. (2013). Long-term dynamics of CA1 hippocampal place codes. Nature Neuroscience, 16(3), 264-6. doi: 10.1038/nn.3329 
bioRxiv preprint doi: https://doi.org/10.1101/2021.02.07.430172; this version posted February 8, 2021. The copyright holder for this preprint (which was not certified by peer review) is the author/funder, who has granted bioRxiv a license to display the preprint in perpetuity. It is made available under aCC-BY-NC-ND 4.0 International license.

SLOW SEQUENCES IN MOUSE HIPPOCAMPUS

\section{Supplementary figures}



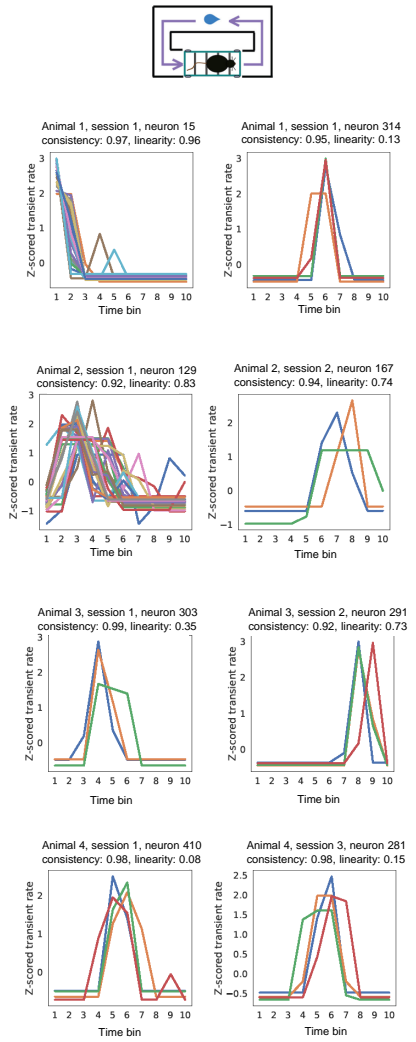
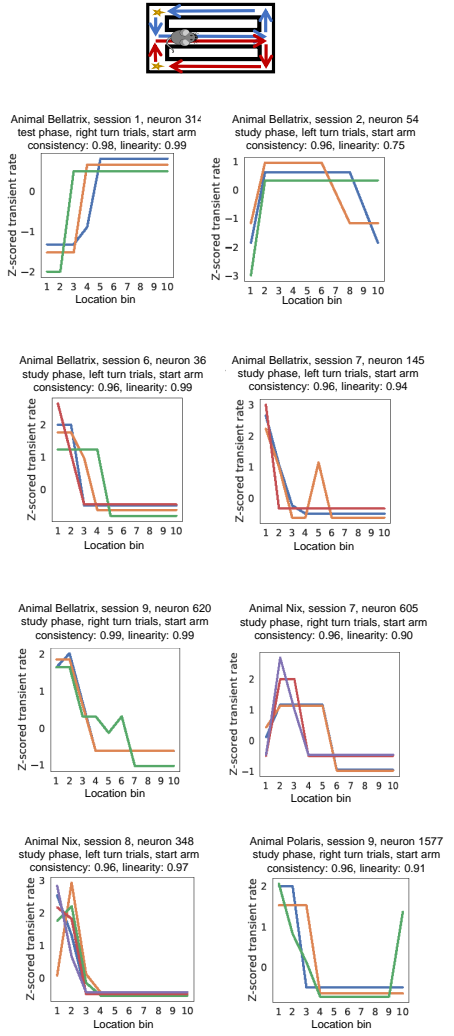

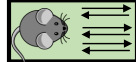
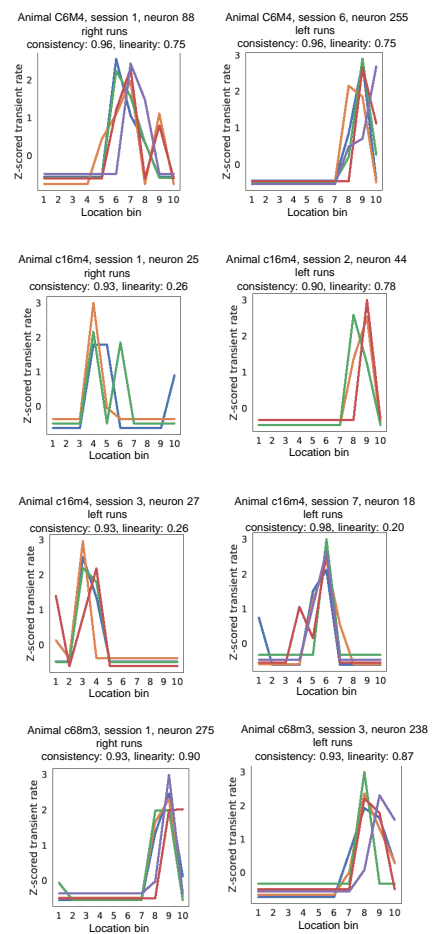

Figure S1. Additional hippocampal neurons that fire consistently within trials across multiple trials. Each line represents the z-scored transient rate of that neuron during a trial. Trials where the neuron is not identified or is inactive are not plotted. 

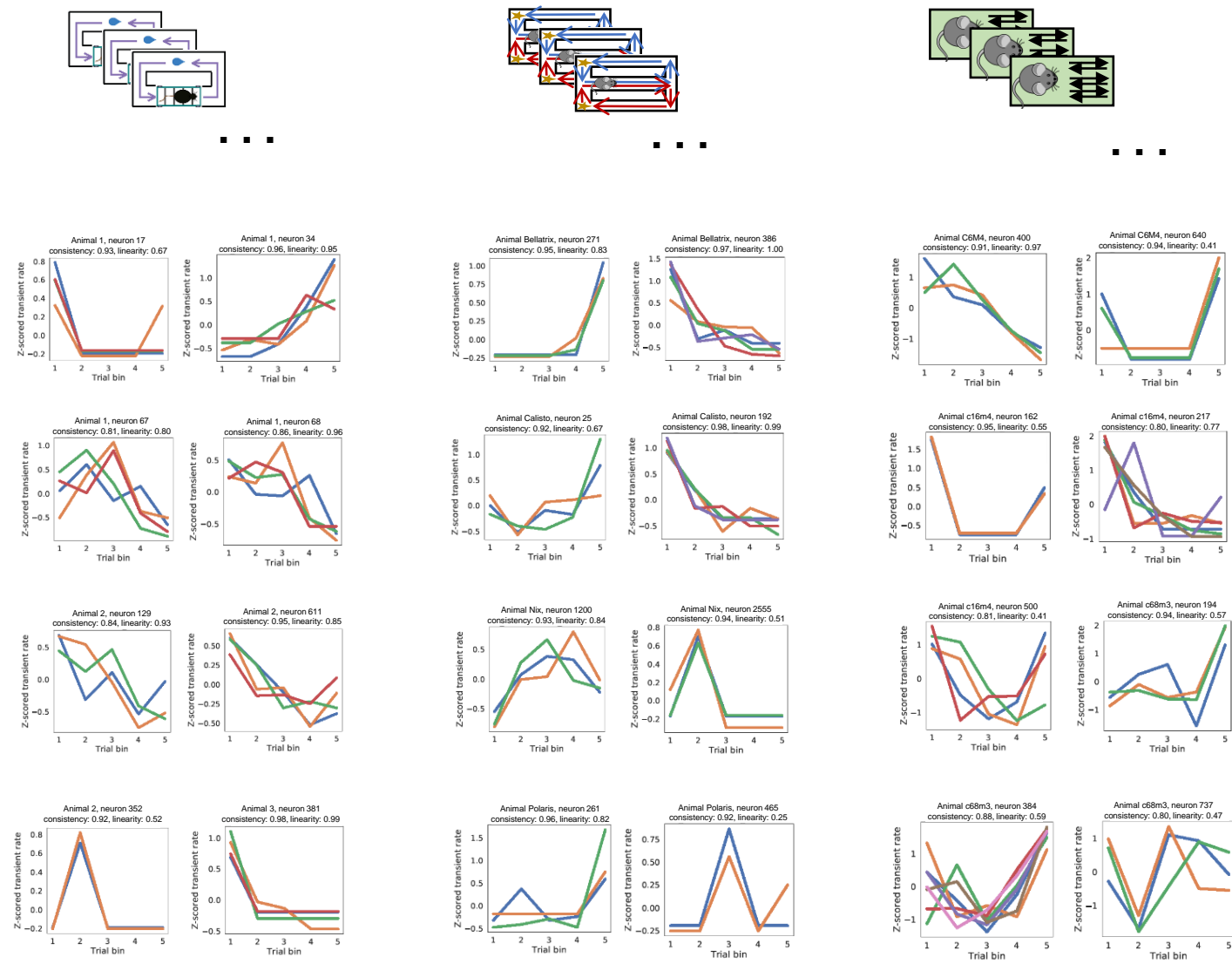

Figure S2. More example hippocampal neurons that fire consistently over specific trials across sessions. Sessions where the neuron is not identified or is inactive are not plotted. 

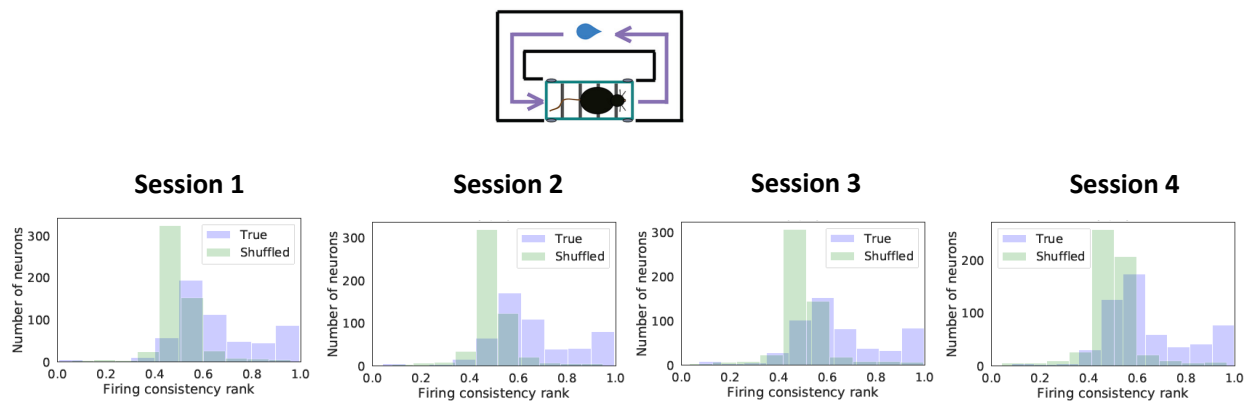

Animal 2
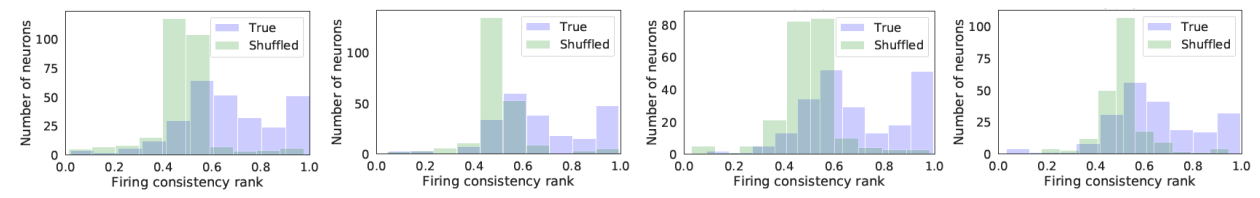

Animal 3
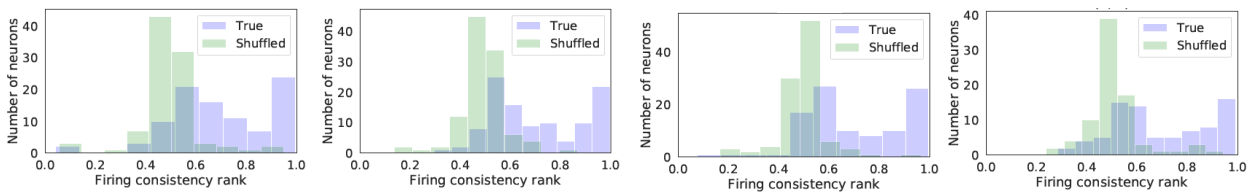

Animal 4
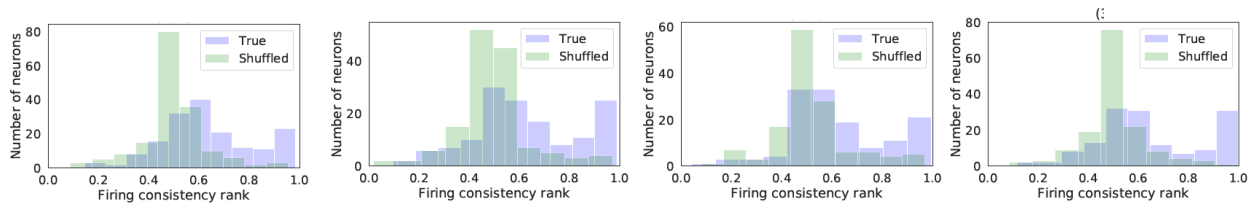

Figure S3. The distribution of the across-trial firing consistency rank for each individual session in the treadmill running task. 

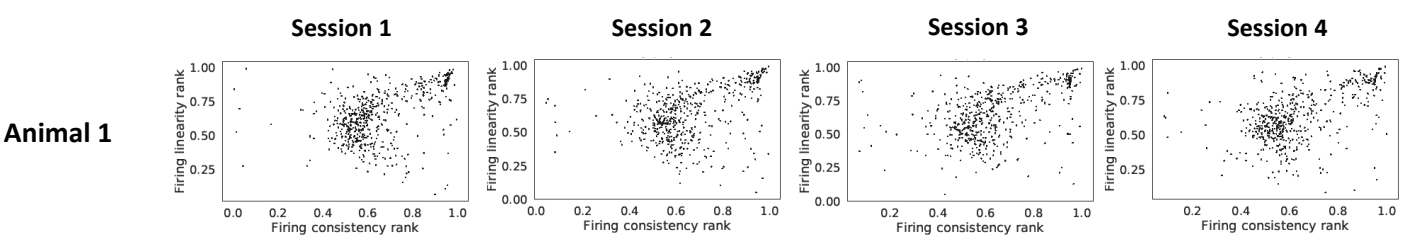

Animal 2
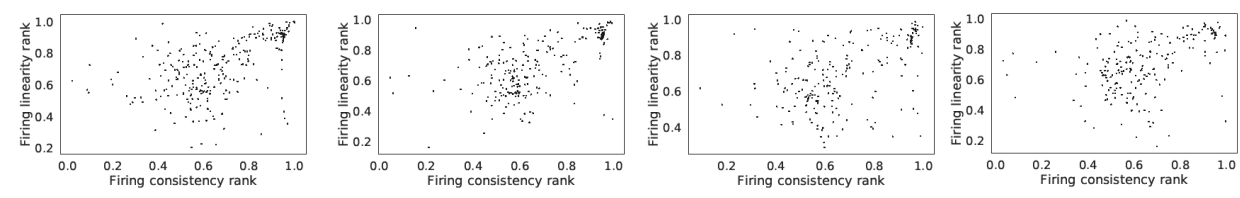

Animal 3
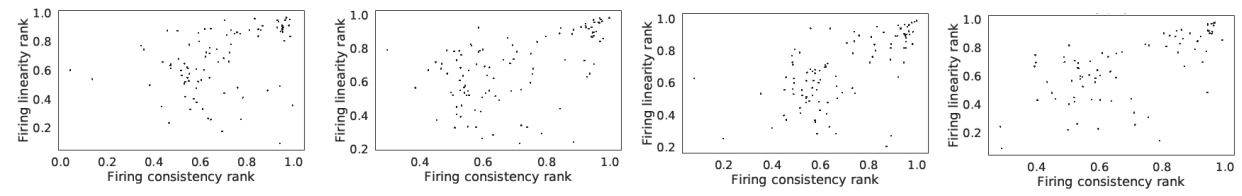

Animal 4
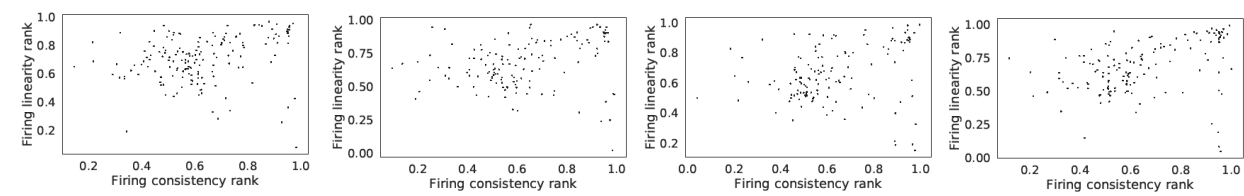

Figure S4. The across-trial firing consistency rank plotted against the across-trial firing linearity rank for each individual session in the treadmill running task. 

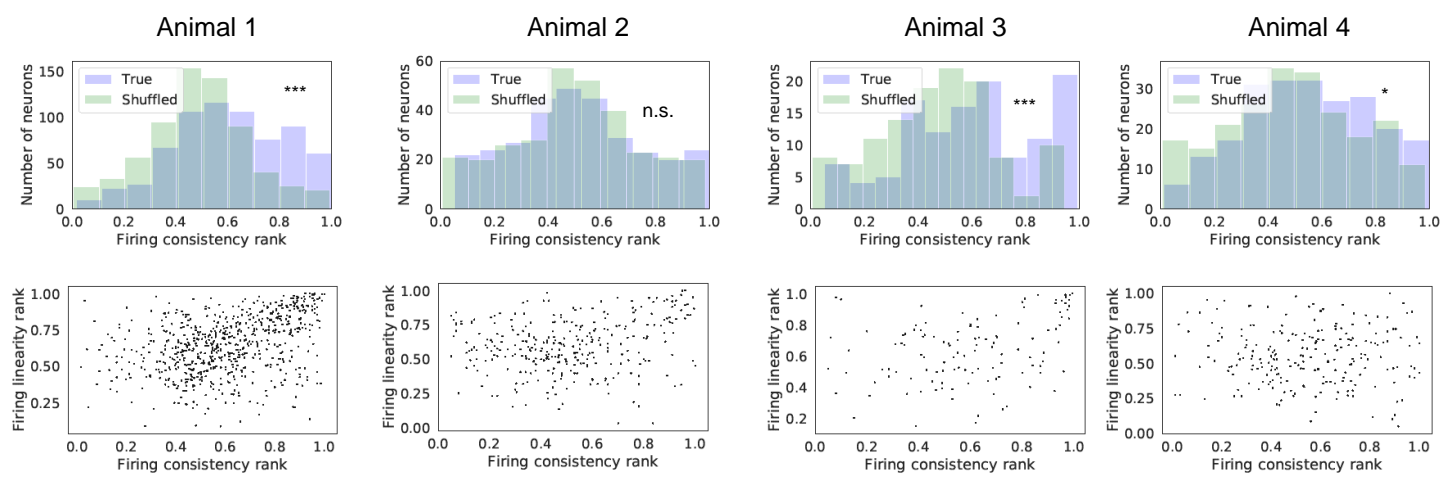

Figure S5. The distribution of the across-session firing consistent rank for real and shuffled data (top) and he joint distribution of the across-session firing consistency rank and firing linearity rank (bottom) for each individual mouse in the treadmill running task 


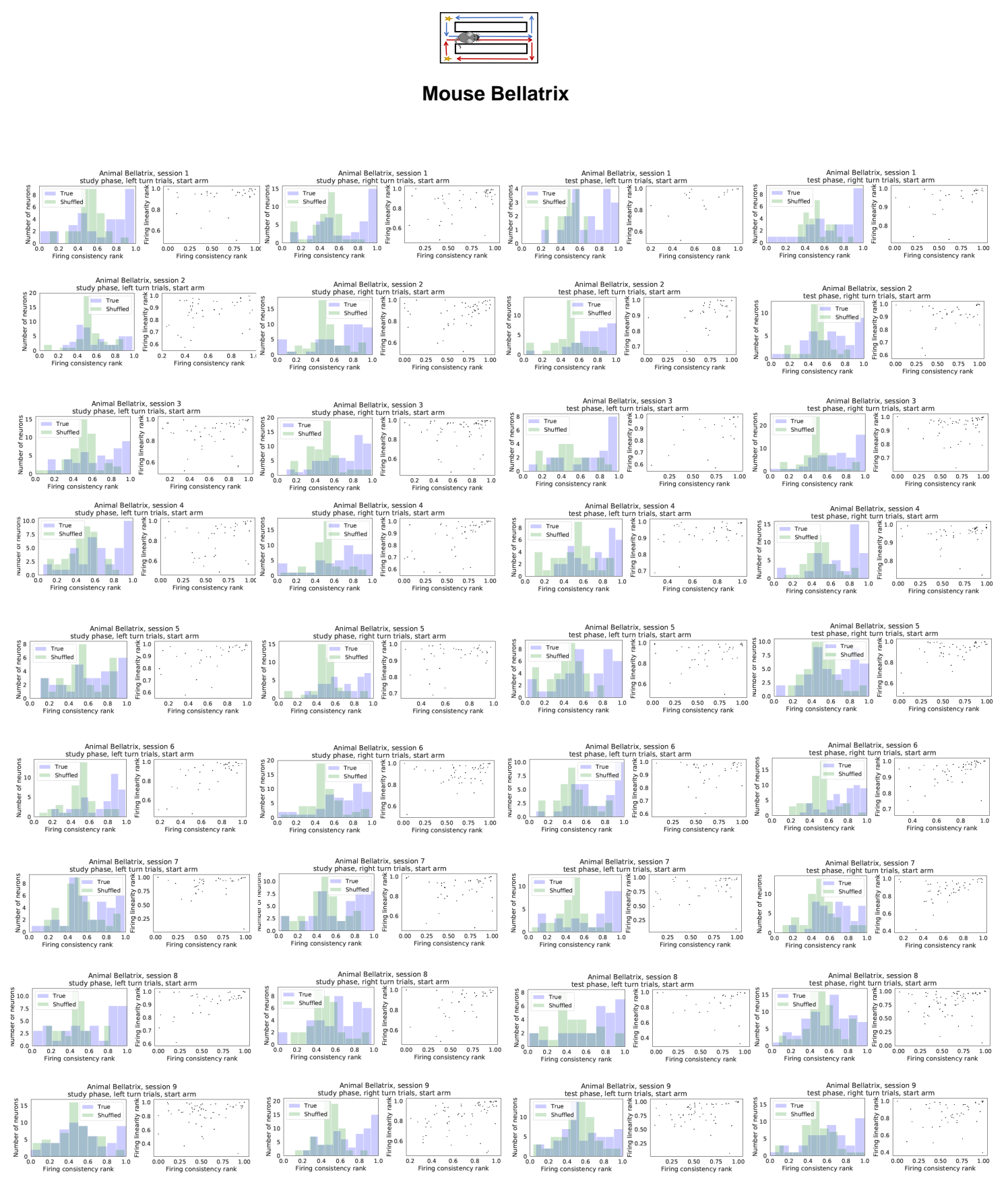

Figure S6. The distribution of the across-trial firing consistent rank for real and shuffled data (left) and the joint distribution of the across-trial firing consistency rank and firing linearity rank (right) for each individual session and trial type in the spatial alternation task. Data for mouse Bellatrix. 


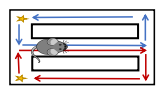

Mouse Calisto
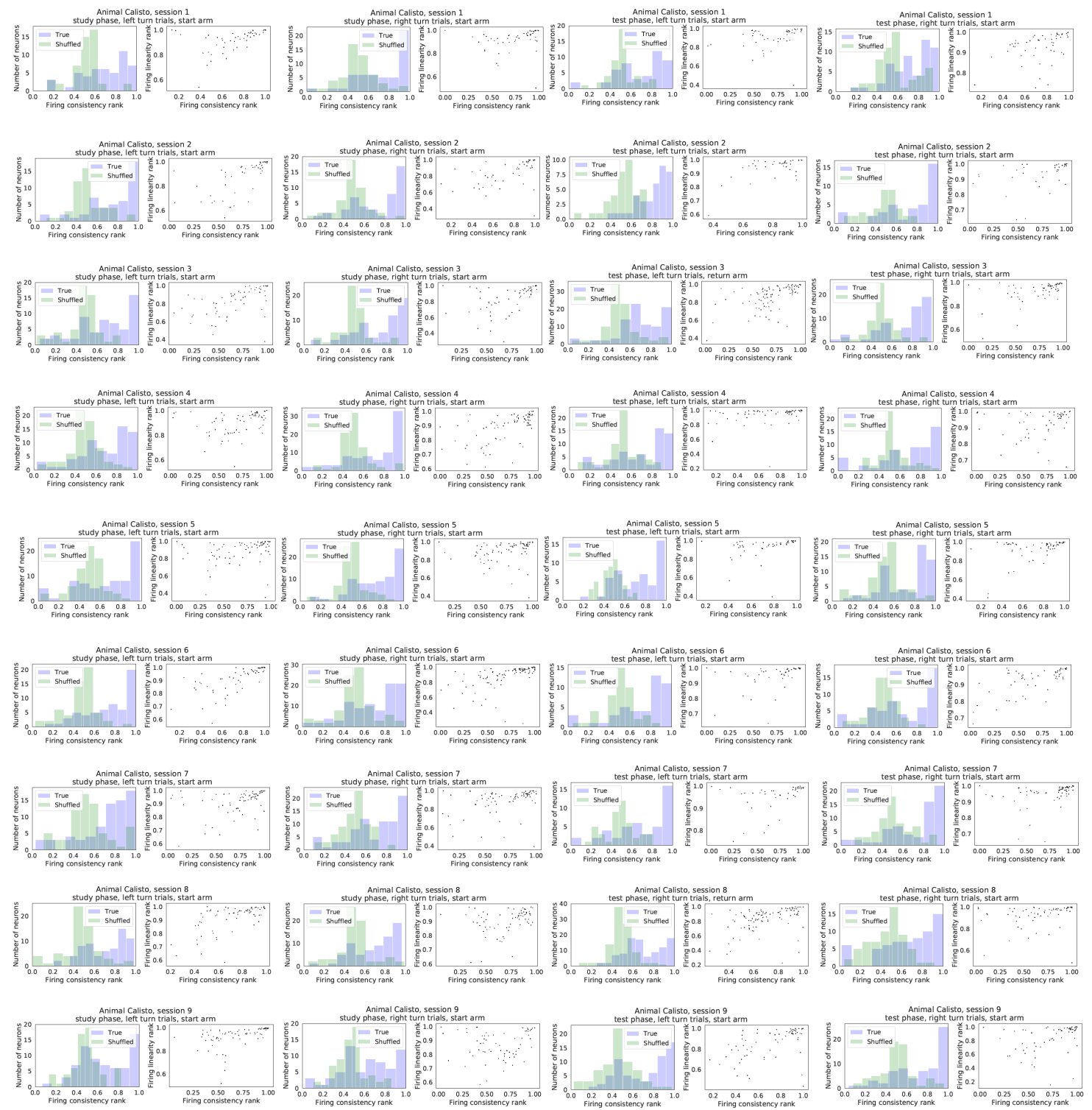

Figure S7. The distribution of the across-trial firing consistent rank for real and shuffled data (left) and the joint distribution of the across-trial firing consistency rank and firing linearity rank (right) for each individual session and trial type in the spatial alternation task. Data for mouse Calisto. 


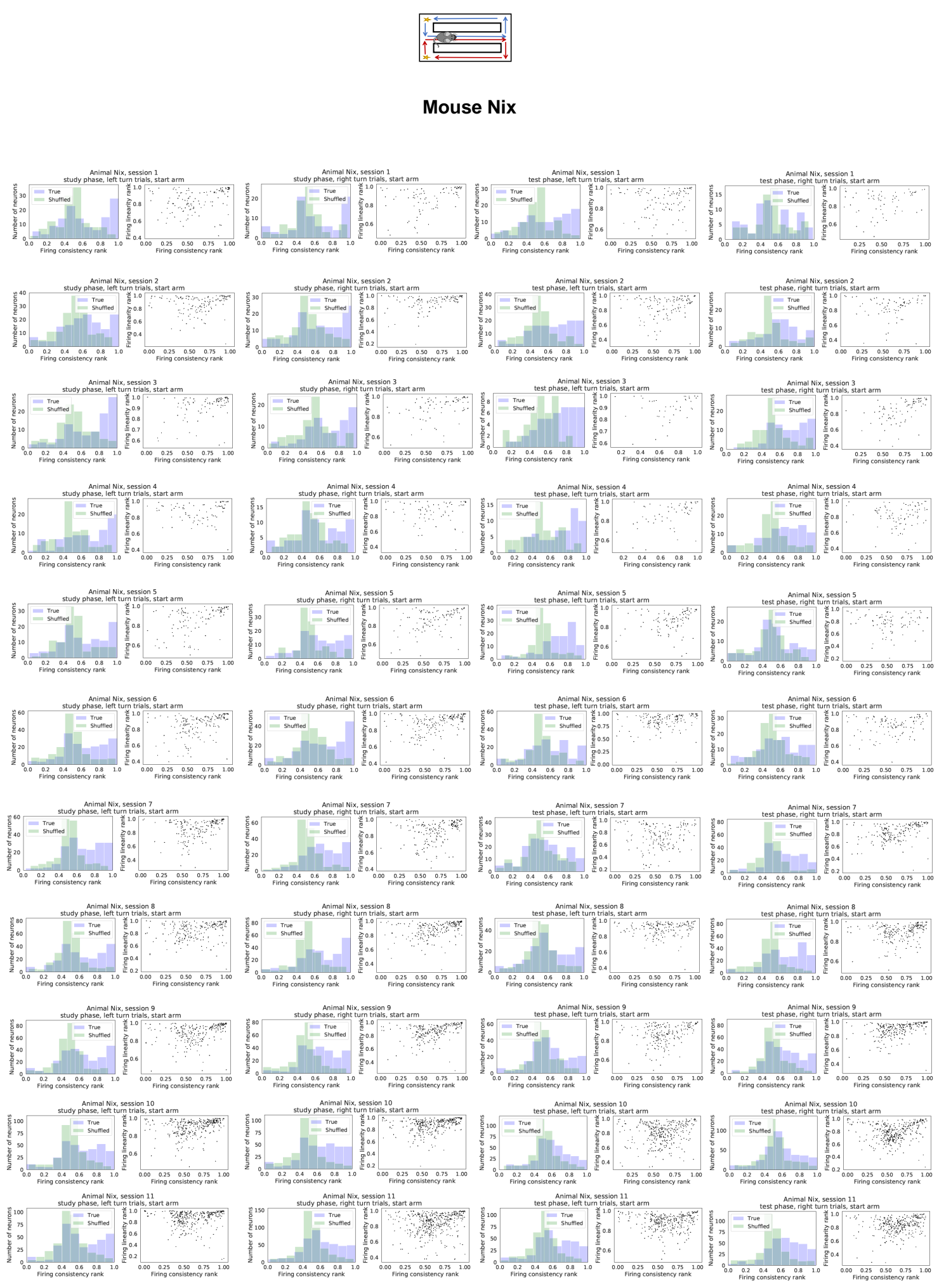

Figure S8. The distribution of the across-trial firing consistent rank for real and shuffled data (left) and the joint distribution of the across-trial firing consistency rank and firing linearity rank (right) for each individual session and trial type in the spatial alternation task. Data for mouse Nix. 


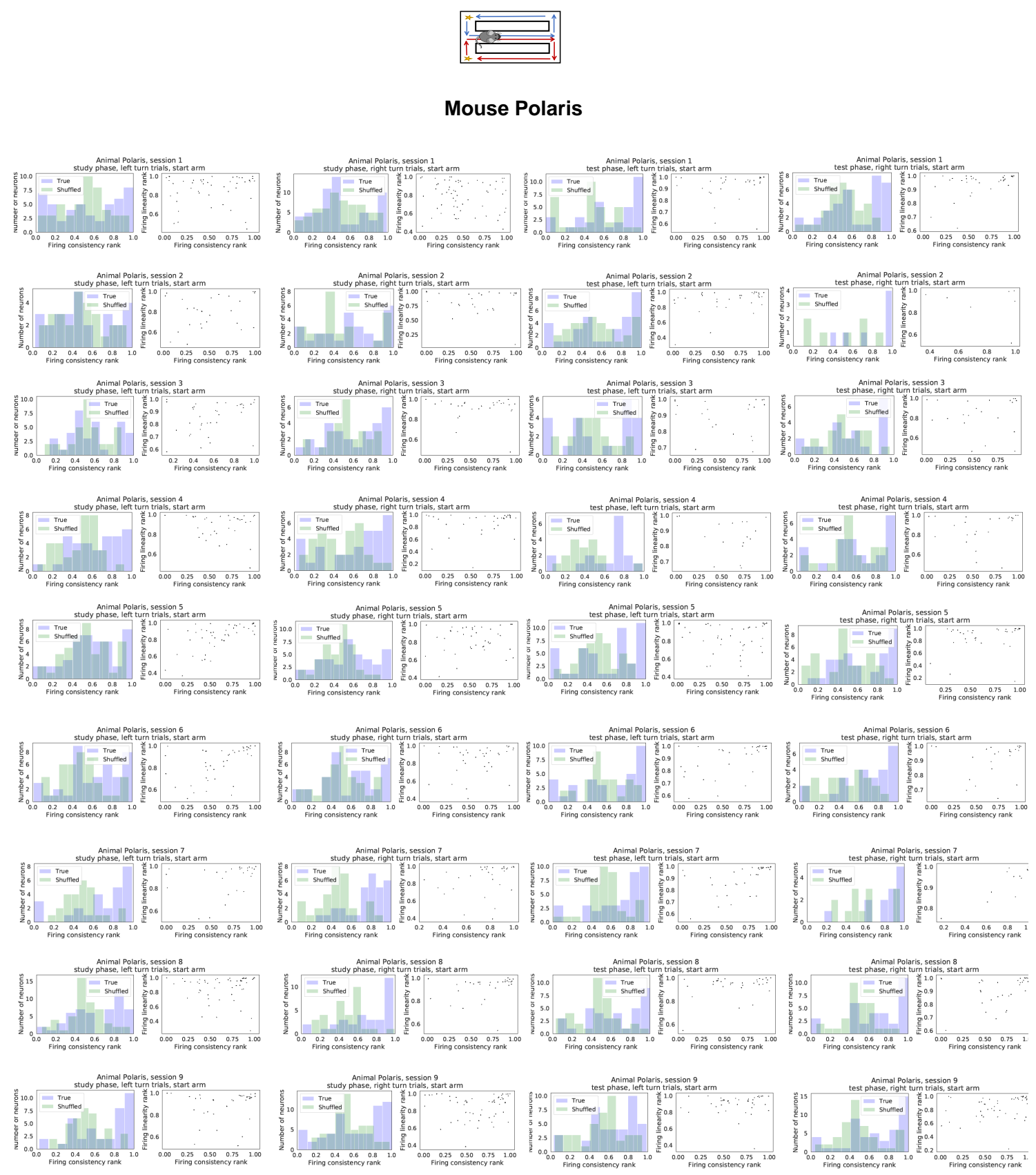

Figure S9. The distribution of the across-trial firing consistent rank for real and shuffled data (left) and the joint distribution of the across-trial firing consistency rank and firing linearity rank (right) for each individual session and trial type in the spatial alternation task. Data for mouse Polaris. 

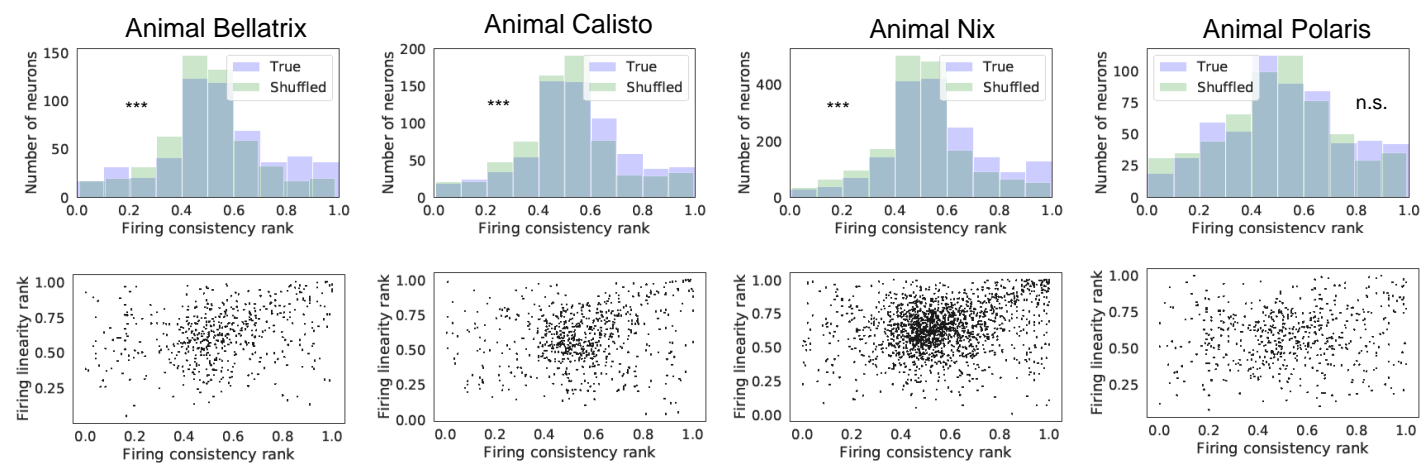

Figure S10. The distribution of the across-session firing consistent rank for real and shuffled data (top) and the joint distribution of the across-session firing consistency rank and firing linearity rank (right) for each individual mouse in the spatial alternation task 


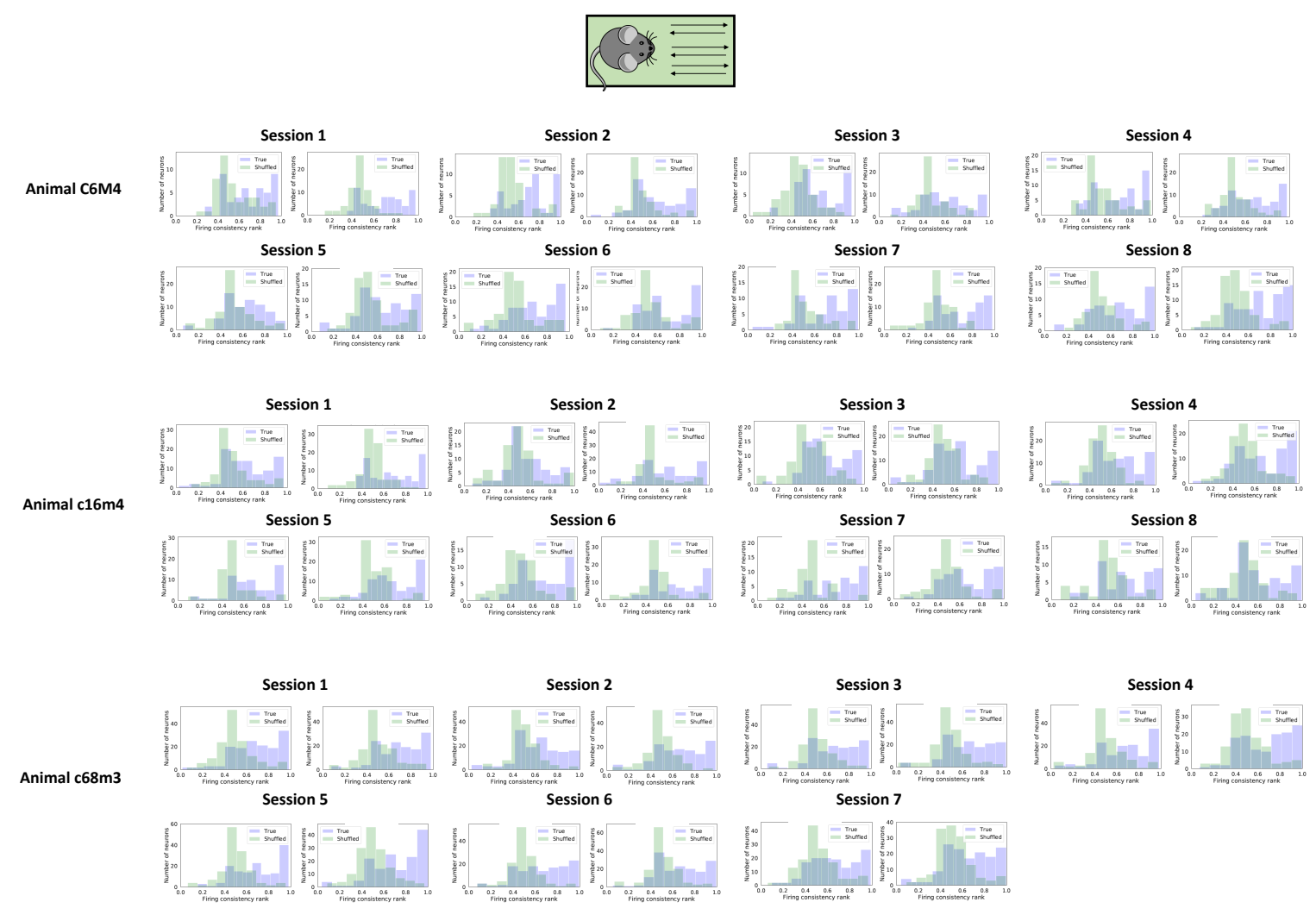

Figure S11. The distribution of the across-trial firing consistency rank for each individual session in the linear track task. Data shown separately for left and right runs 


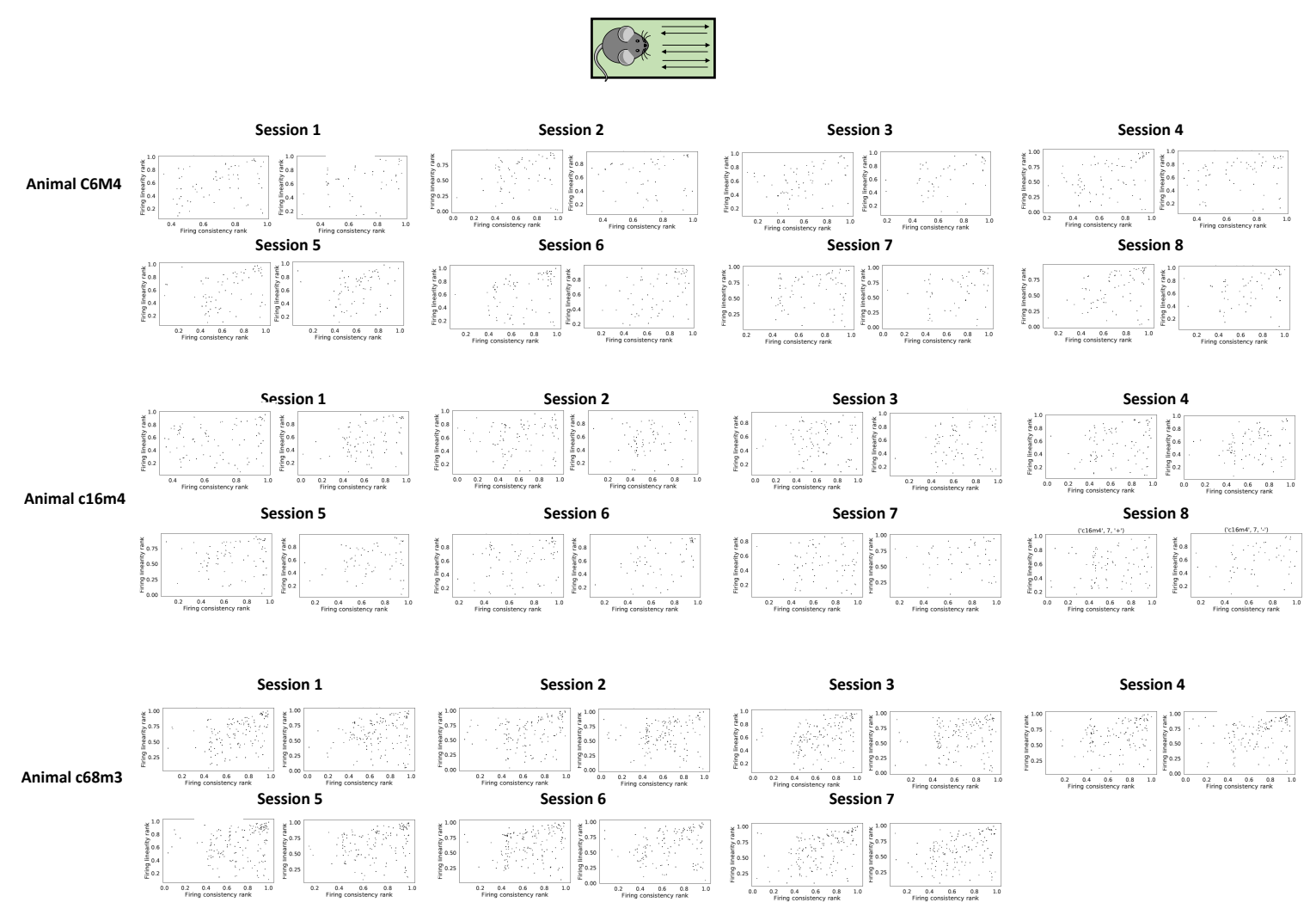

Figure S12. The across-trial firing consistency rank plotted against the across-trial firing linearity rank for each individual session in the linear track task. 

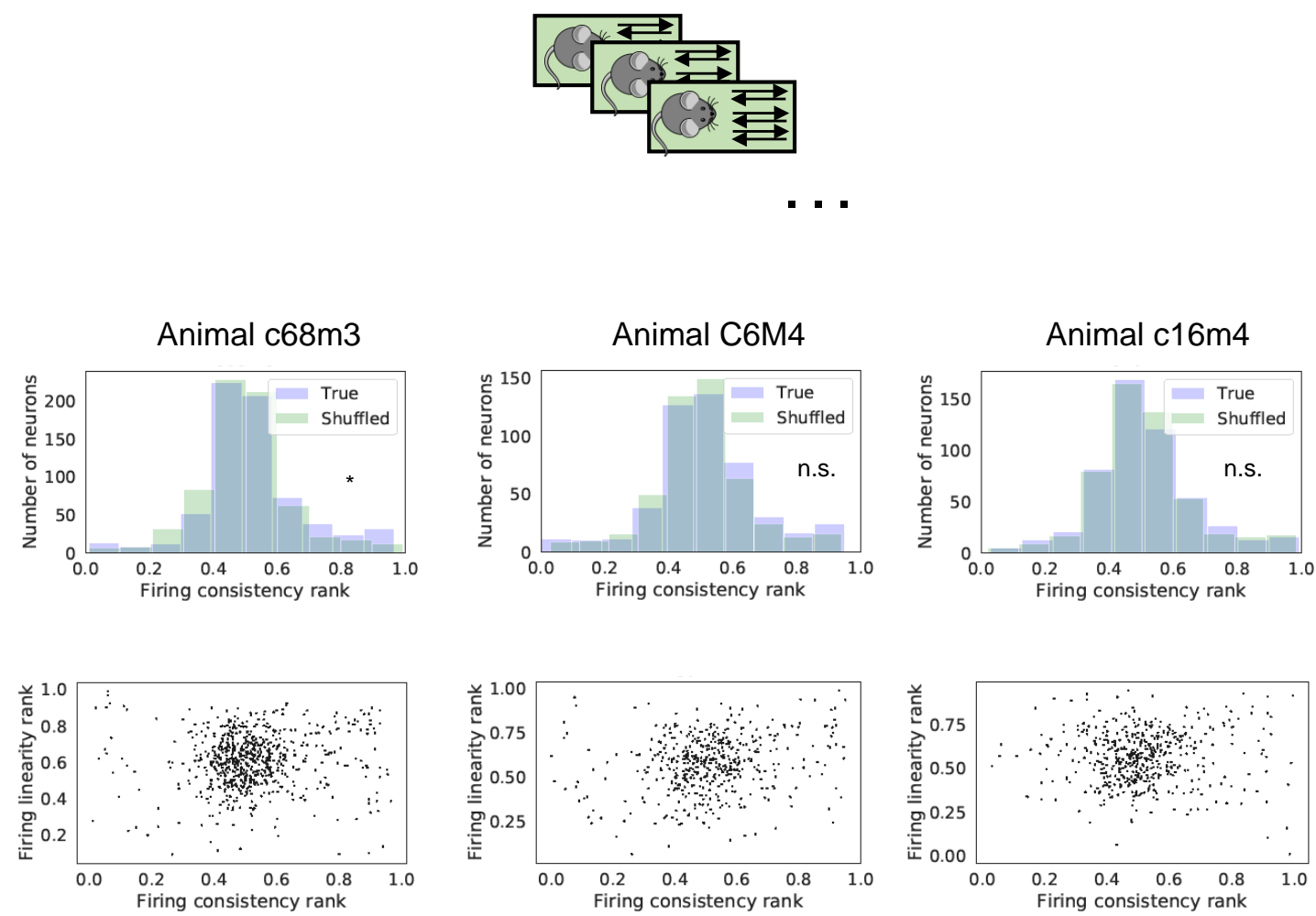

Figure S13. The distribution of the across-session firing consistent rank for real and shuffled data (top) and the joint distribution of the across-session firing consistency rank and the firing linearity rank (bottom) for each individual mouse in the linear track task 
a.
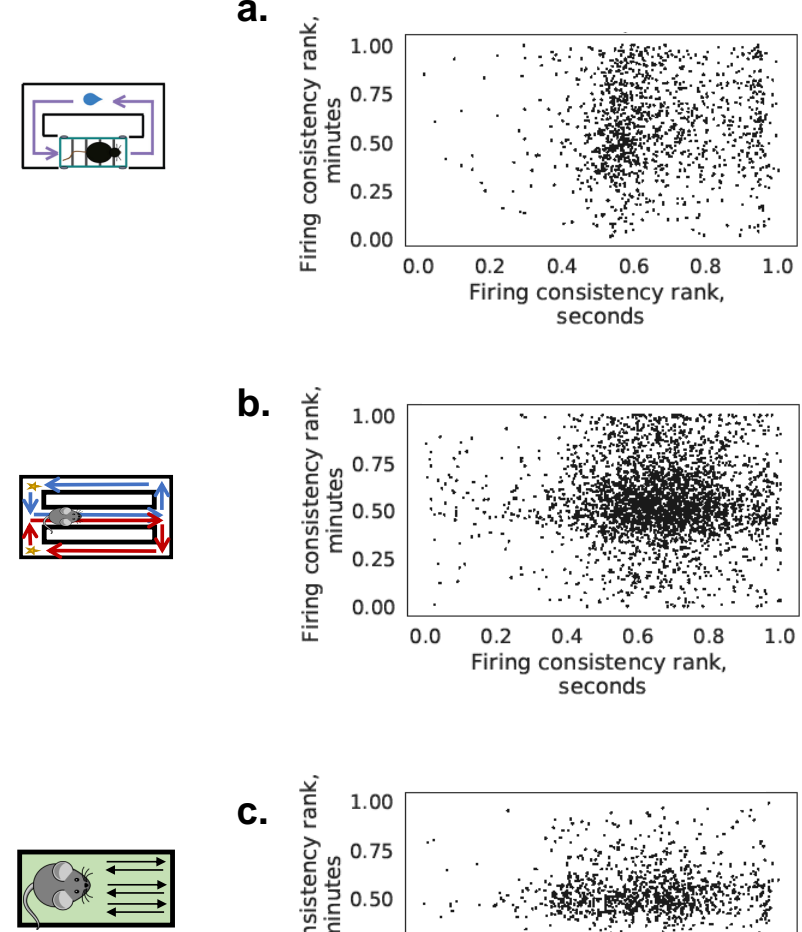

b.

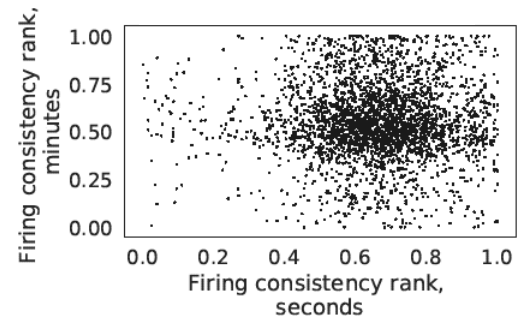

c.

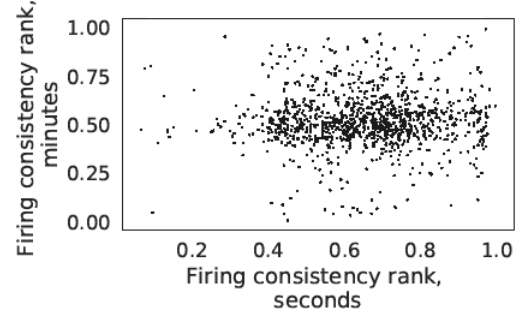

d.

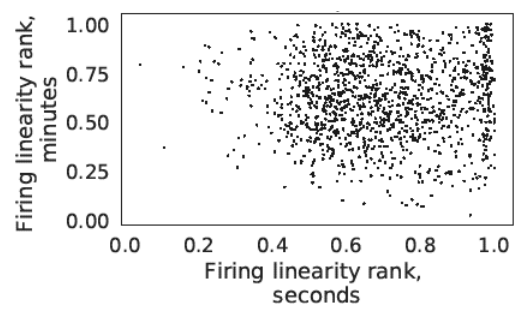

e.

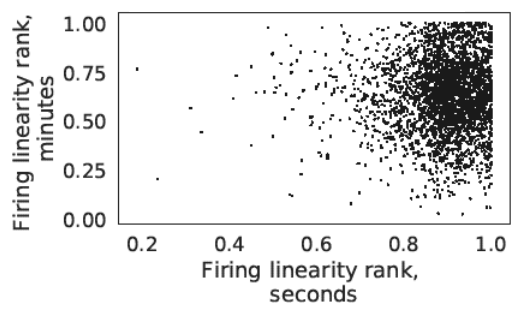

f.

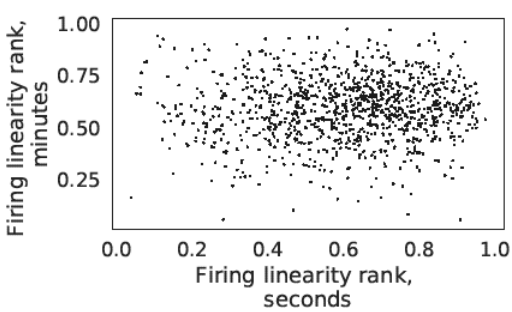

Figure S14. The firing consistency and linearity ranks are not meaningfully correlated when comparing within trials timescale (seconds) to within sessions timescale (minutes). The correlation between the consistency rank across trials and sessions for the treadmill running (a), spatial alternation (b) and linear track (c) experiments. The same for the firing linearity rank (d-f). Kendall's $\tau: \mathbf{a}: \tau=0.05, p=0.008, n=1241$. b: $\tau=-0.008, p=$ $0.488, n=2999$. c: $\tau=0.02, p=0.293, n=1098 . \mathbf{d}: \tau=-0.002, p=0.912, n=1241$. $\mathbf{e}:$ $\tau=0.002, p=0.867, n=2999$. f: $\tau=0.03, p=0.09, n=1058$. 
Animal 1
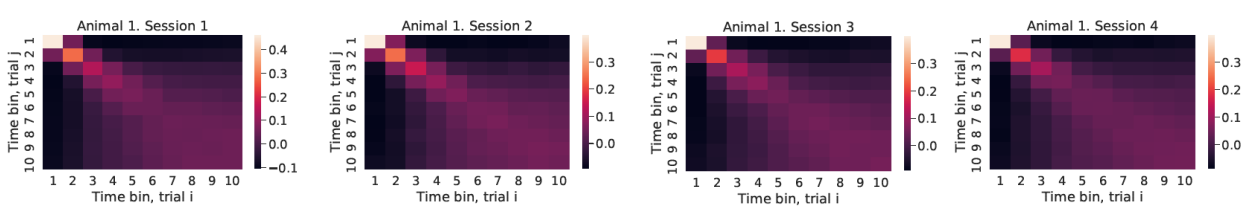

Animal 2
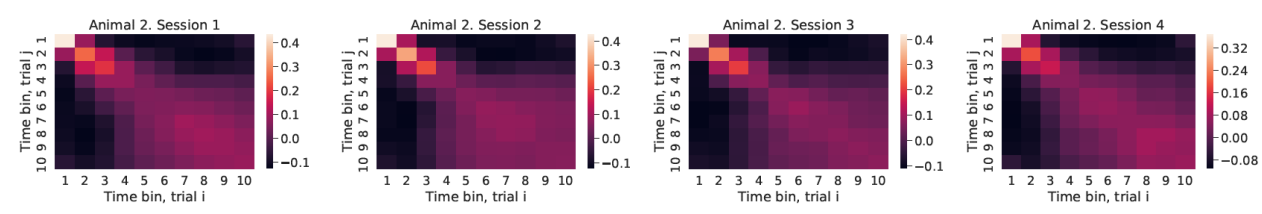

Animal 3
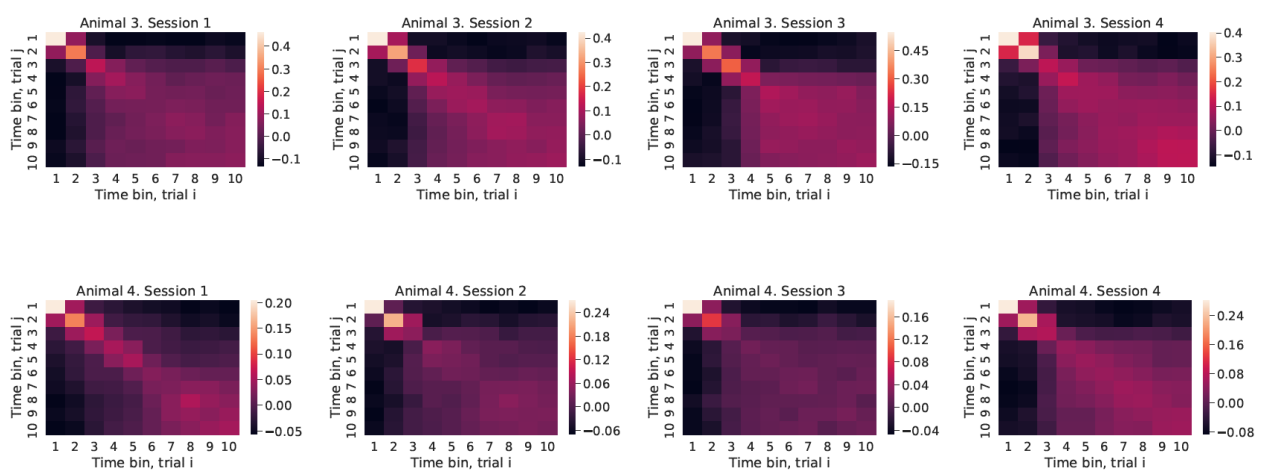

Figure S15. The cross-trial correlations for each individual session in the treadmill running task 

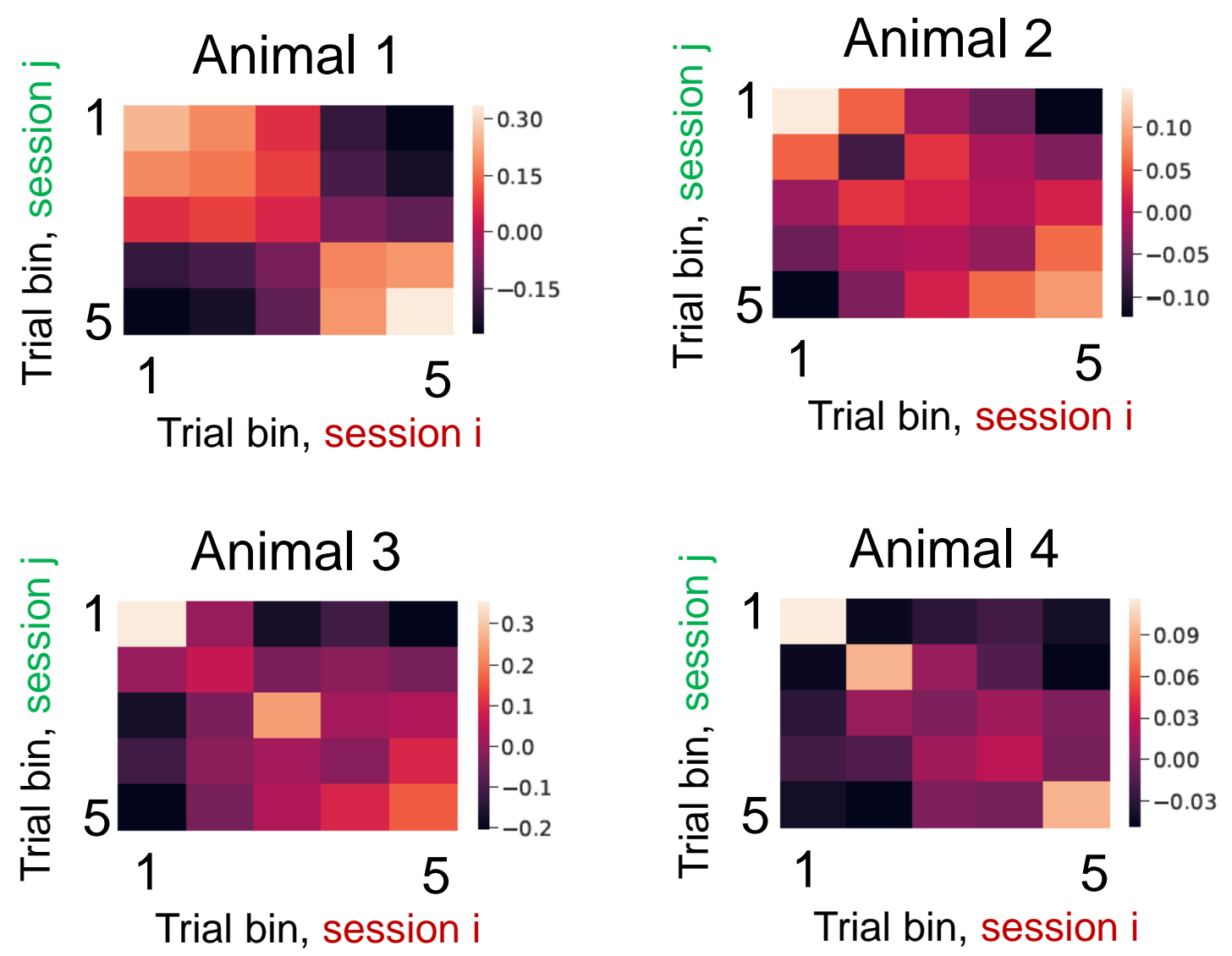

Figure S16. The cross-session correlations for each individual mouse in the treadmill running task 
bioRxiv preprint doi: https://doi.org/10.1101/2021.02.07.430172; this version posted February 8, 2021. The copyright holder for this preprint (which was not certified by peer review) is the author/funder, who has granted bioRxiv a license to display the preprint in perpetuity. It is made available under aCC-BY-NC-ND 4.0 International license.

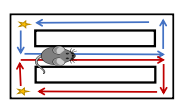

\section{Mouse Bellatrix}
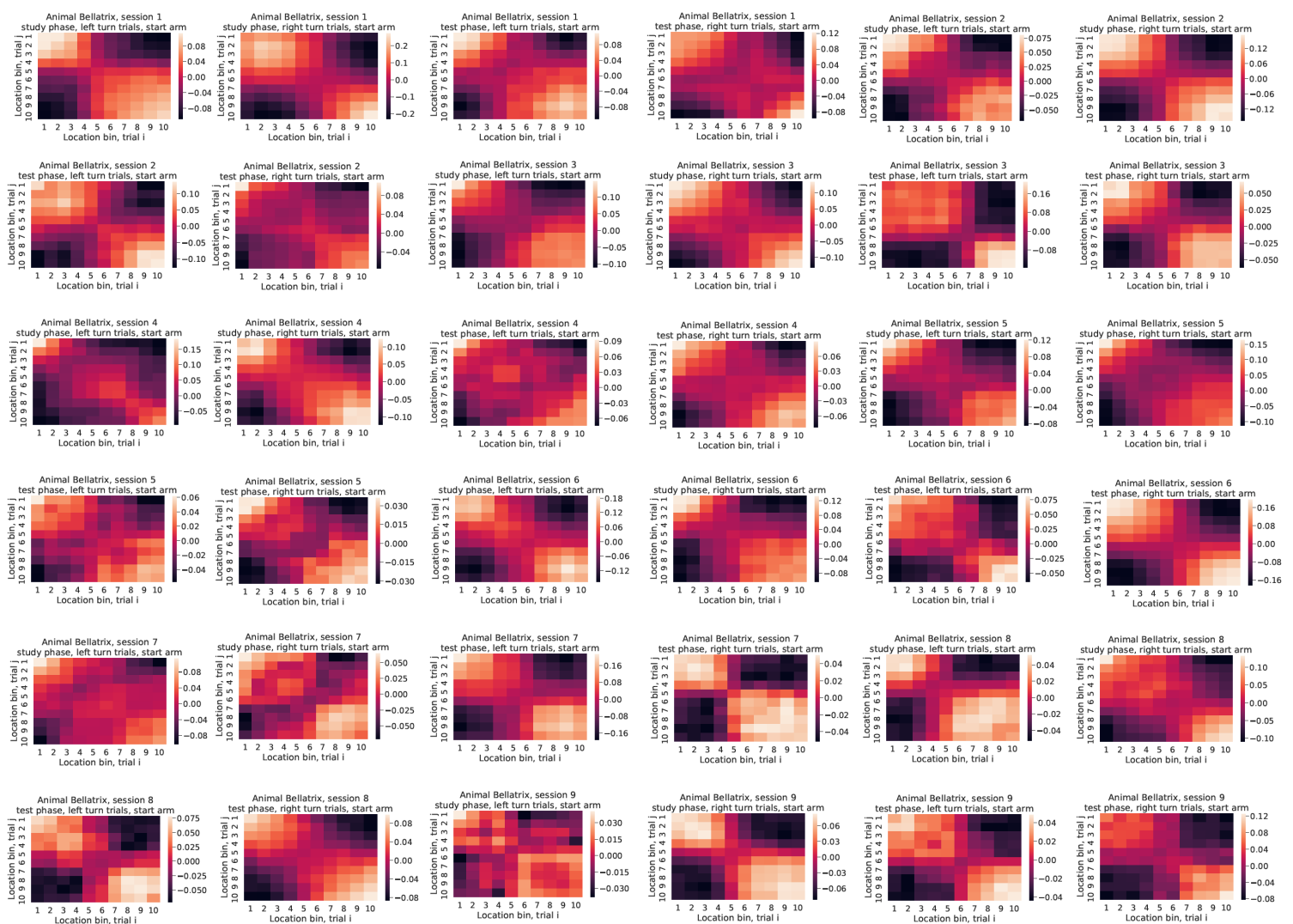

Figure S17. The cross-trial correlations for each individual session, task phase and turn direction in the spatial alternation task. Data for mouse Bellatrix. 
bioRxiv preprint doi: https://doi.org/10.1101/2021.02.07.430172; this version posted February 8, 2021. The copyright holder for this preprint (which was not certified by peer review) is the author/funder, who has granted bioRxiv a license to display the preprint in perpetuity. It is made available under aCC-BY-NC-ND 4.0 International license.

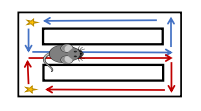

\section{Mouse Calisto}
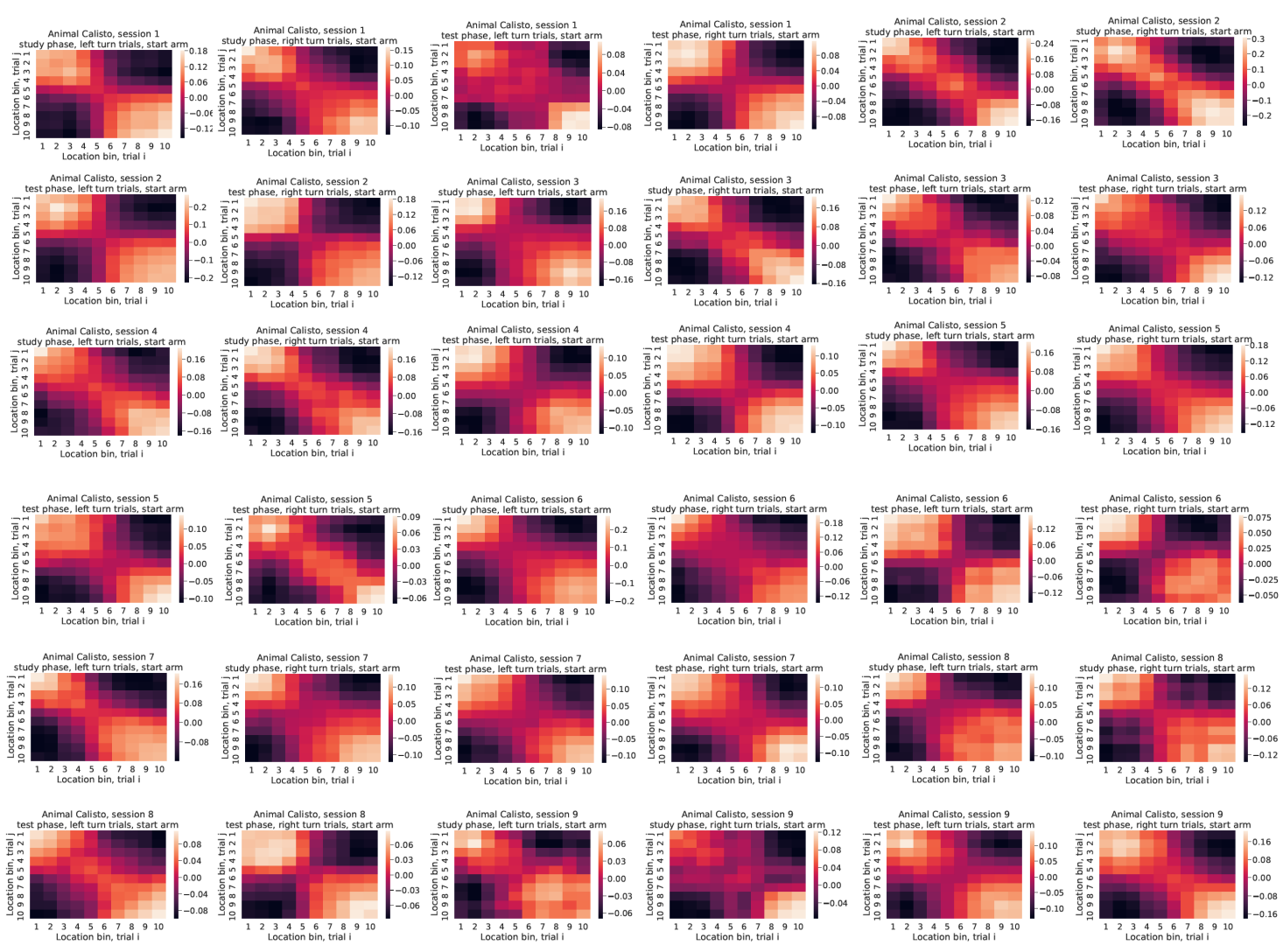

Figure S18. The cross-trial correlations for each individual session, task phase and turn direction in the spatial alternation task. Data for mouse Calisto. 
bioRxiv preprint doi: https://doi.org/10.1101/2021.02.07.430172; this version posted February 8, 2021. The copyright holder for this preprint (which was not certified by peer review) is the author/funder, who has granted bioRxiv a license to display the preprint in perpetuity. It is made available under aCC-BY-NC-ND 4.0 International license.

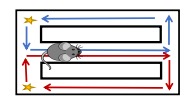

\section{Mouse Nix}
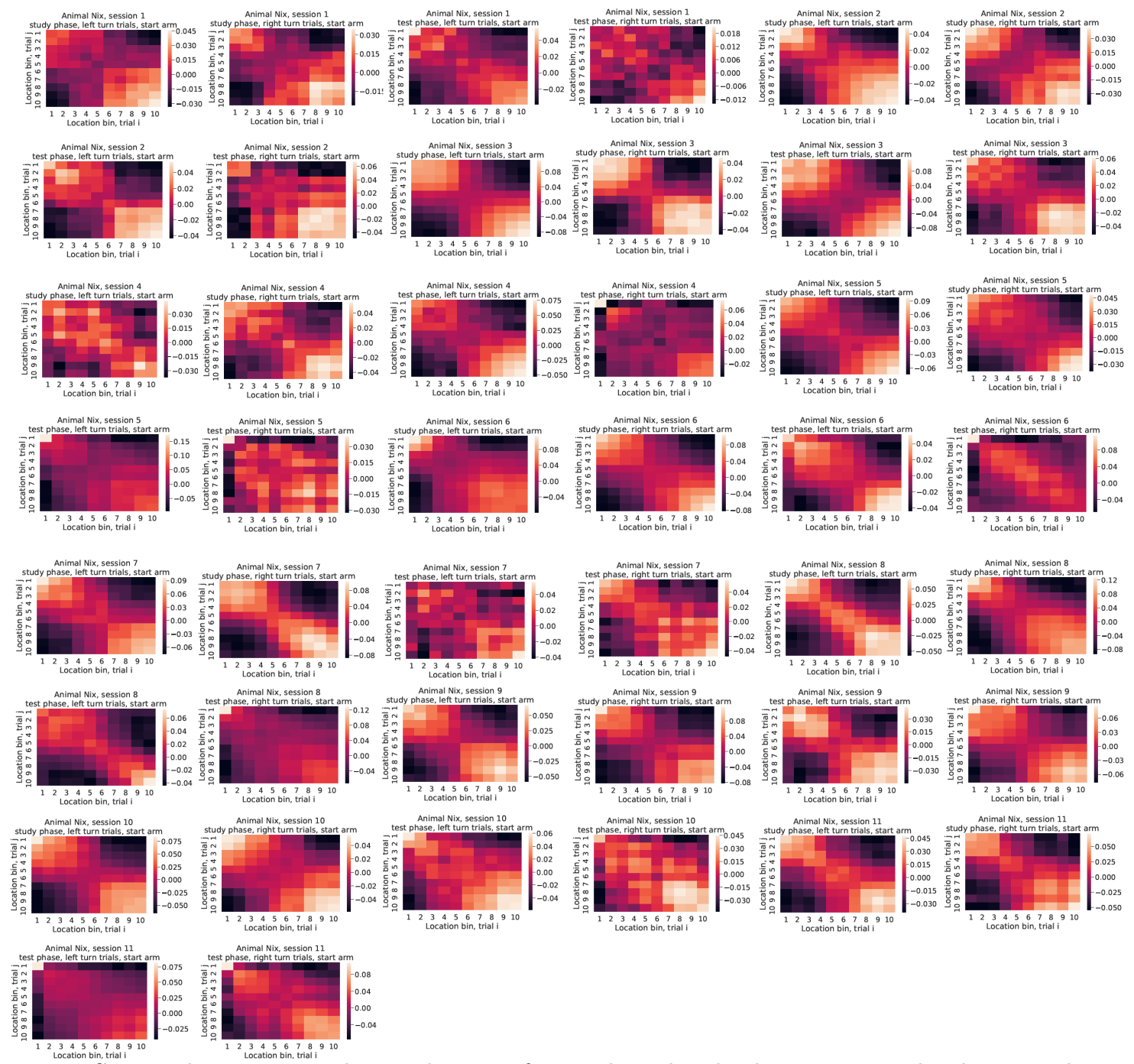

Figure S19. The cross-trial correlations for each individual session, task phase and turn direction in the spatial alternation task. Data for mouse Nix. 
bioRxiv preprint doi: https://doi.org/10.1101/2021.02.07.430172; this version posted February 8, 2021. The copyright holder for this preprint (which was not certified by peer review) is the author/funder, who has granted bioRxiv a license to display the preprint in perpetuity. It is made available under aCC-BY-NC-ND 4.0 International license.

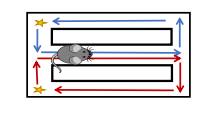

\section{Mouse Polaris}
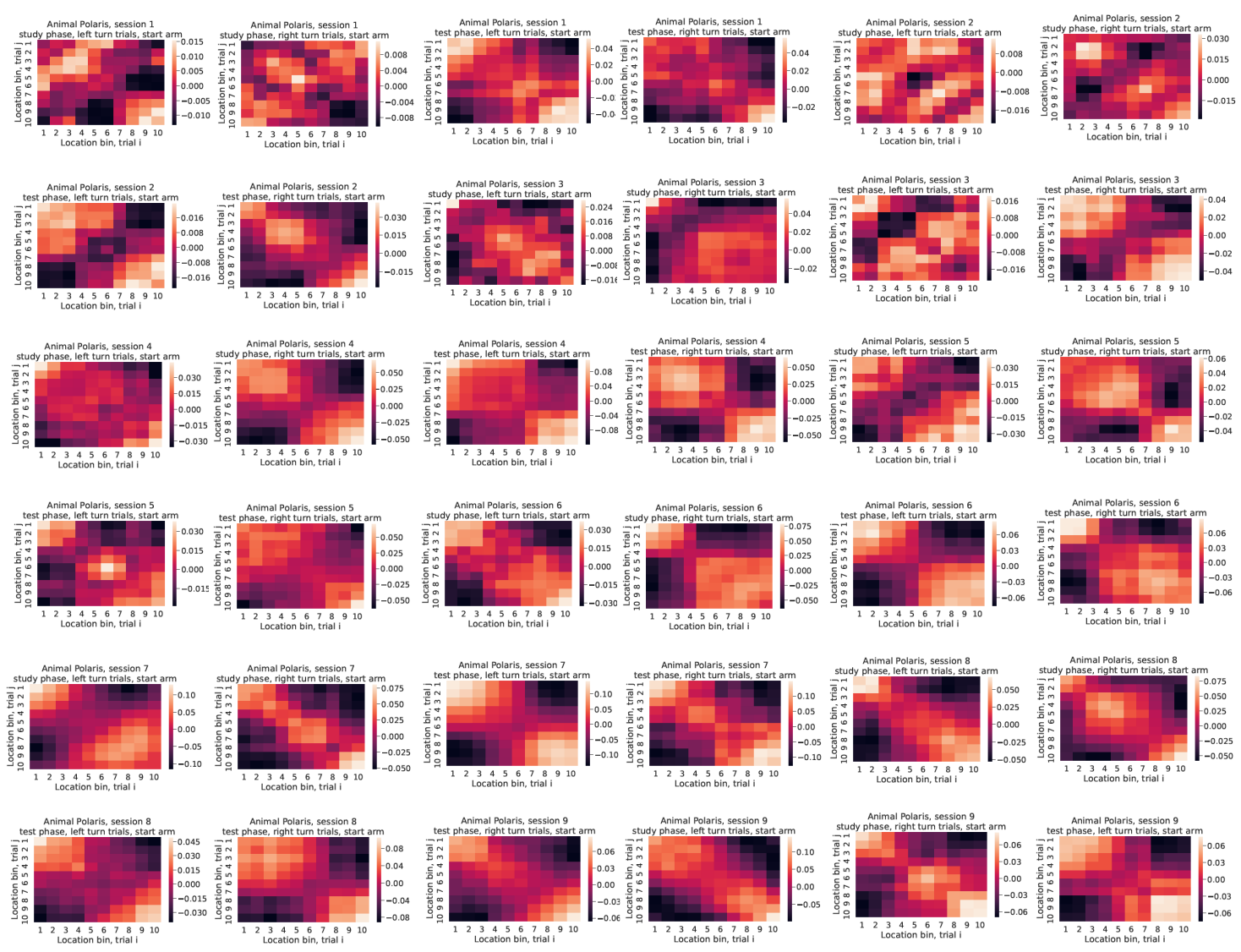

Figure S20. The cross-trial correlations for each individual session, task phase and turn direction in the spatial alternation task. Data for mouse Polaris. 


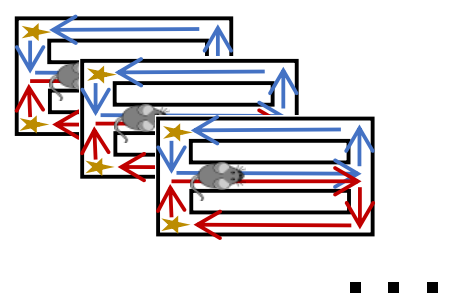

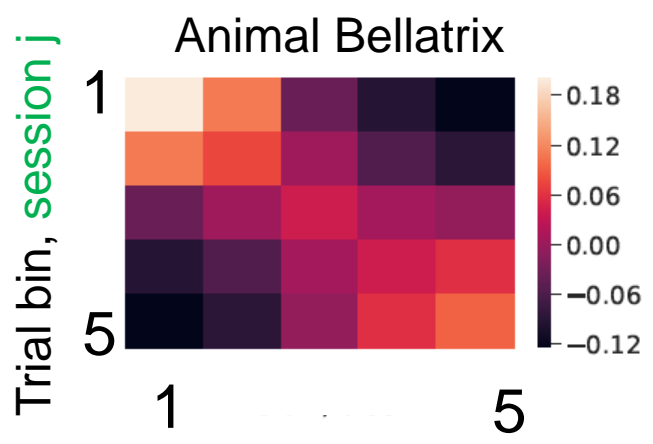

Trial bin, session i

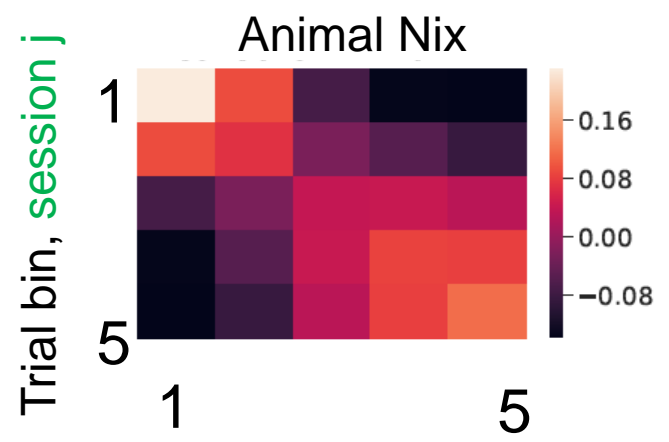

Trial bin, session i
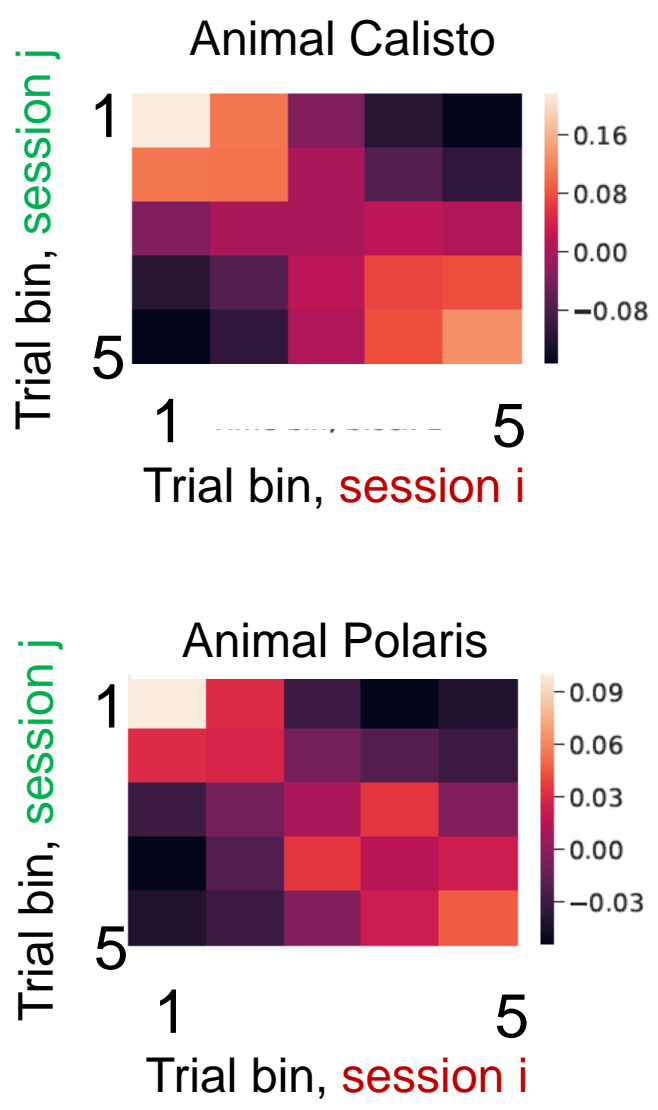

Figure S21. The cross-session correlations for each individual mouse in the spatial alternation task 
bioRxiv preprint doi: https://doi.org/10.1101/2021.02.07.430172; this version posted February 8, 2021. The copyright holder for this preprint (which was not certified by peer review) is the author/funder, who has granted bioRxiv a license to display the preprint in perpetuity. It is made available under aCC-BY-NC-ND 4.0 International license.

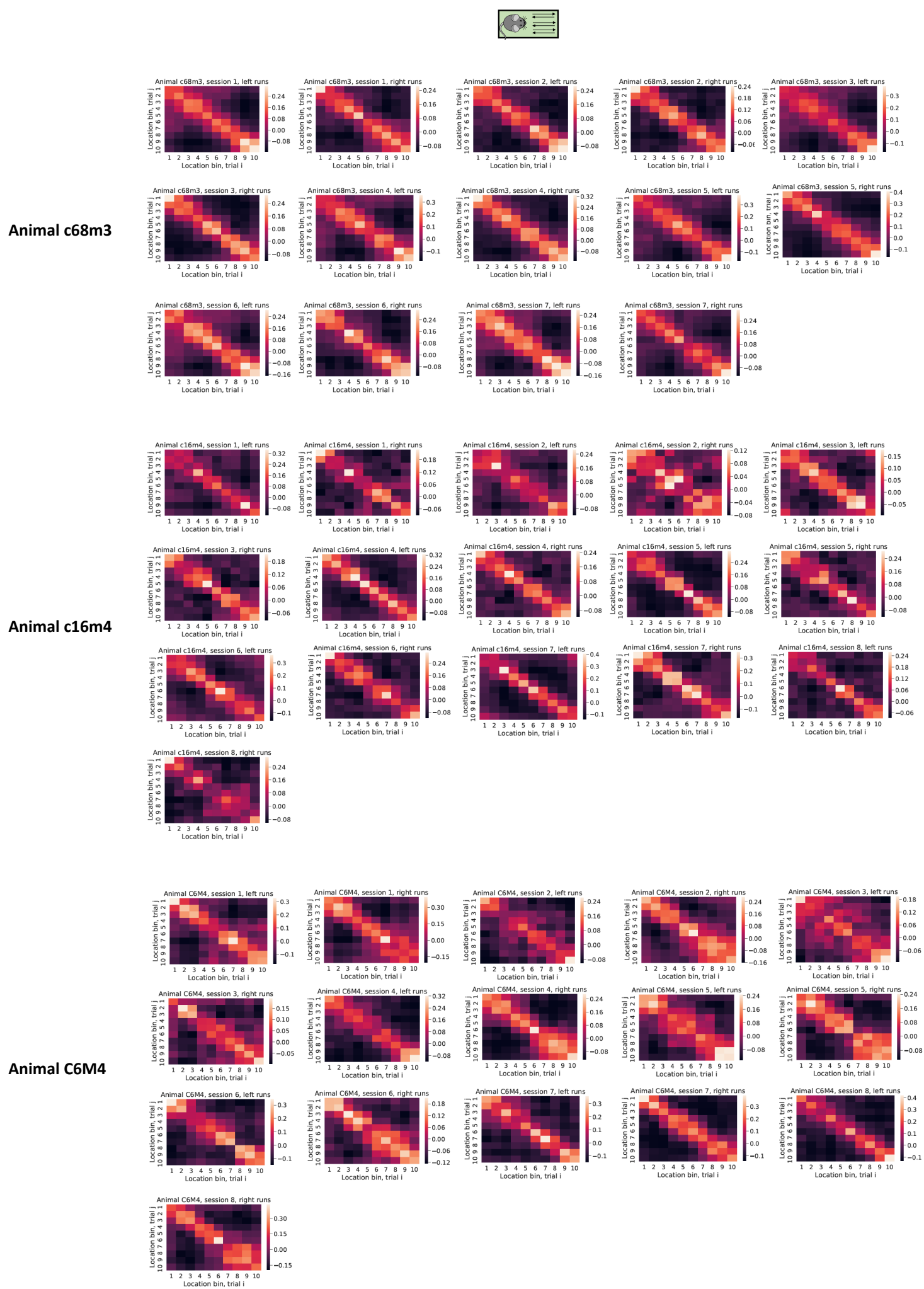

Figure S22. The cross-trial correlations for each individual session and running direction in the linear track task 

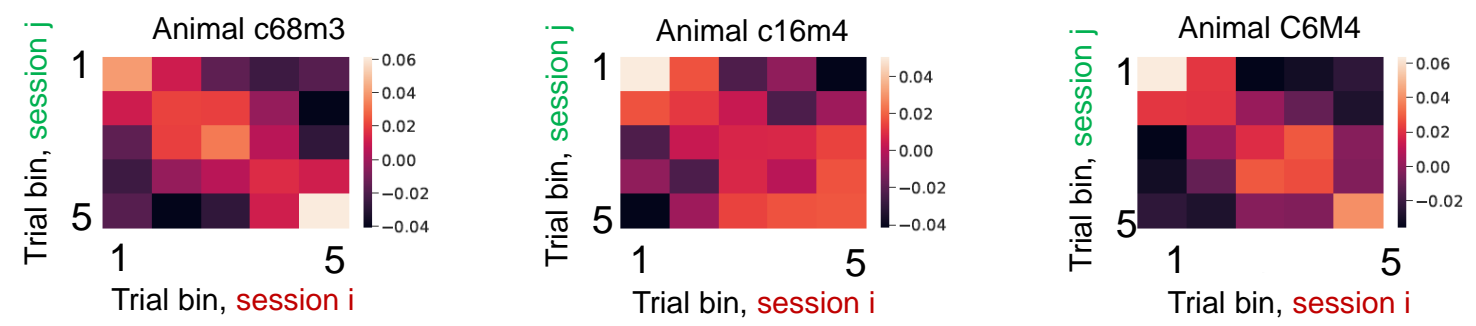

Figure S23. The cross-session correlations for each individual mouse in the linear track task 